\title{
Short telomere syndromes cause a primary T cell immunodeficiency
}

\author{
Christa L. Wagner, ${ }^{1}$ Vidya Sagar Hanumanthu, ${ }^{1}$ C. Conover Talbot Jr., ${ }^{2}$ Roshini S. Abraham, ${ }^{3}$ David Hamm, ${ }^{4}$ Dustin L. Gable, ${ }^{1}$ \\ Christopher G. Kanakry, ${ }^{1}$ Carolyn D. Applegate, ${ }^{5}$ Janet Siliciano, ${ }^{6}$ J. Brooks Jackson, ${ }^{7}$ Stephen Desiderio, ${ }^{2,8,9}$ Jonathan K. Alder, ${ }^{1}$ \\ Leo Luznik, ${ }^{1,8}$ and Mary Armanios ${ }^{1,5,7,8,9}$ \\ 1Department of Oncology and ${ }^{2}$ Institute for Basic Biomedical Sciences, Johns Hopkins University School of Medicine, Baltimore, Maryland, USA. ${ }^{3}$ Department of Laboratory Medicine and Pathology, \\ Mayo Clinic, Rochester, Minnesota, USA. ${ }^{4}$ Adaptive Biotechnologies, Seattle, Washington, USA. ${ }^{5}$ McKusick-Nathans Institute of Genetic Medicine, ${ }^{6}$ Department of Medicine, ${ }^{7}$ Department of Pathology, \\ ${ }^{8}$ Sidney Kimmel Comprehensive Cancer Center, and ${ }^{9}$ Department of Molecular Biology and Genetics, Johns Hopkins University School of Medicine, Baltimore, Maryland, USA.
}

\begin{abstract}
The mechanisms that drive $\mathrm{T}$ cell aging are not understood. We report that children and adult telomerase mutation carriers with short telomere length (TL) develop a T cell immunodeficiency that can manifest in the absence of bone marrow failure and causes life-threatening opportunistic infections. Mutation carriers shared T cell-aging phenotypes seen in adults 5 decades older, including depleted naive T cells, increased apoptosis, and restricted T cell repertoire. T cell receptor excision circles (TRECs) were also undetectable or low, suggesting that newborn screening may identify individuals with germline telomere maintenance defects. Telomerase-null mice with short TL showed defects throughout T cell development, including increased apoptosis of stimulated thymocytes, their intrathymic precursors, in addition to depleted hematopoietic reserves. When we examined the transcriptional programs of T cells from telomerase mutation carriers, we found they diverged from older adults with normal TL. Short telomere T cells upregulated DNA damage and intrinsic apoptosis pathways, while older adult T cells upregulated extrinsic apoptosis pathways and programmed cell death 1 (PD-1) expression. T cells from mice with short TL also showed an active DNA-damage response, in contrast with old WT mice, despite their shared propensity to apoptosis. Our data suggest there are TL-dependent and TL-independent mechanisms that differentially contribute to distinct molecular programs of T cell apoptosis with aging.
\end{abstract}

\section{Introduction}

$\mathrm{T}$ cell number and function decline with age; this age-dependent program, at times referred to as $\mathrm{T}$ cell immunosenescence, contributes to increased morbidity $(1,2)$. Several $\mathrm{T}$ cell phenotypic changes occur with aging; these include a depletion of naive T cells that is associated with thymic involution, a decline in T cell-proliferative capacity, and a progressive restriction of the $\mathrm{T}$ cell repertoire. While these age-associated changes are well described, the genetic and molecular programs that drive $\mathrm{T}$ cell aging remain poorly understood $(1,2)$.

Telomere length (TL) is a heritable trait; it is determined by the parental TL as well as common polymorphisms near the telomerase and telomere maintenance genes (3-5). Telomeres shorten with age, and at critical cellular thresholds, short telomeres become dysfunctional, signaling a p53-related DNA-damage response that provokes senescence and apoptosis (6-8). While telomere shortening is universally acquired with aging, there are clinically relevant thresholds in the human population that are associated with telomere-mediated disease (9). Importantly,

Conflict of interest: $\mathrm{DH}$ is an employee of Adaptive Biotechnologies, a company that specializes in T cell receptor sequencing.

License: Copyright 2018, American Society for Clinical Investigation.

Submitted: February 5, 2018; Accepted: August 28, 2018.

Reference information: J Clin Invest. 2018;128(12):5222-5234.

https://doi.org/10.1172/JCI120216. although TL shortens in T cells with aging, the causal contribution of short telomeres to the human T cell-aging phenotype has not been established. The T cell-replicative potential has been hypothesized to be sensitive to telomere reserves, since $\mathrm{T}$ cell memory needs to be maintained through adulthood (10). To minimize telomere attrition with replication, $\mathrm{T}$ cell receptor (TCR) activation upregulates telomerase activity (10). However, this increase in telomerase activity does not entirely offset telomere shortening $(3,11)$, and the replicative demands throughout life cause $\mathrm{T}$ cells to have the highest rate of telomere attrition with age relative to other leukocyte subsets $(11,12)$.

Mendelian disorders provide a clinically relevant context for distinguishing the causal contribution of short telomeres from correlative changes that occur with aging. In humans, short telomeres manifest in a monogenic disease spectrum known as short telomere syndromes (13). They are most commonly caused by lossof-function mutations in the genes encoding the telomerase core enzyme components: the telomerase reverse transcriptase, TERT, and the telomerase RNA TR (also known as TERC) (9). TL is the primary determinant of disease severity in these patients (9). Children diagnosed with short telomere syndromes have more severe telomere defects than adults and develop bone marrow failure that is at times recognized in the disorder dyskeratosis congenita (14-16). Adults with milder TL defects develop lung disease, both idiopathic pulmonary fibrosis (IPF) and emphysema (9). The prevalence of these lung disorders and the high proportion of IPF 
patients who carry mutant telomerase genes make the short telomere syndromes the most common premature aging disorders (13). Recognizing short telomere syndrome patients at the bedside is critical, since they develop severe toxicities to otherwise tolerated therapies $(9,13)$. They are also exquisitely sensitive to ionizing radiation, similar to patients with other DNA double-strand repair syndromes (17), making conventional protocols for hematopoietic stem cell transplantation highly toxic and fatal (18).

Here, we report a primary and potentially fatal $\mathrm{T}$ cell immunodeficiency in young patients with short telomere syndromes, its overall prevalence, and its association with recurrent patterns of opportunistic infections. Some affected patients lacked the classic hallmarks of telomere-mediated disease, including bone marrow failure. We show that $\mathrm{T}$ cells from short telomere syndrome patients show a premature aging phenotype, but in contrast with $\mathrm{T}$ cells from older adults (OA) with normal TL, have a distinct molecular program related to the DNA-damage response and intrinsic apoptosis. Our data identify abnormally short telomeres as sufficient to drive $\mathrm{T}$ cell aging, but suggest that additional TL-independent mechanisms promote $\mathrm{T}$ cell aging in individuals with normal TL.

\section{Results}

Telomerase mutation carriers develop a severe and potentially fatal $T$ cell immunodeficiency. We noted a high prevalence of opportunistic infections in a Johns Hopkins-based cohort of short telomere syndrome patients. Among 28 patients under the age of 60 who were evaluated from 2005 to 2015, 9 (32\%) developed opportunistic infections associated with $\mathrm{T}$ cell immunodeficiency (Table 1 and Figure $1 \mathrm{~A})$. Their absolute $\mathrm{CD} 4^{+} \mathrm{T}$ cell counts and, where available, absolute $\mathrm{CD} 8^{+} \mathrm{T}$ cell counts were abnormally low (Table 1). For more than half the patients ( 5 of $9,55 \%$ ), there was no clinical evidence of bone marrow failure at the time of diagnosis (Table 1). The infections were most commonly related to herpes viruses: varicella zoster (VZV) and cytomegalovirus (CMV) (6 of 9, 66\%, Table 1 and Figure 1, A-E). In cases in which CMV caused endorgan disease, either encephalitis or pneumonitis, the infection was fatal ( 3 of 3, Table 1 and Figure 1, A, D, and E). To characterize the immunodeficiency of short telomere syndromes, we examined immune indices for 17 mutation carriers, including 10 individuals who carried germline mutations in telomerase genes, but had no history of infection, and found the most common abnormalities were CD4 lymphopenia and low IgM levels (Figure 1F). These observations indicate that a combined immunodeficiency may be a first manifestation of short telomere syndromes. This immunodeficiency can appear in adults in the absence of bone marrow failure and predispose to life-threatening opportunistic infections.

Telomerase mutation carriers show severe depletion of naive $T$ cells. Since short telomeres are acquired with aging, we tested whether short telomere syndrome-mediated immunodeficiency resembles the $\mathrm{T}$ cell-aging phenotype. We designed a 3-way comparison of young patients who carried mutations in telomerase genes (hereafter referred to as short telomere [ST], mean age, 21 years), young healthy controls (YC) (mean age, 26 years), and healthy OA (mean age, 73 years) (Figure 2A and Supplemental Table 1; supplemental material available online with this article; https://doi.org/10.1172/JCI120216DS1). YC and OA had normal age-adjusted TL, near the 50th percentile (Figure 2, A and B). On the other hand, ST patients had abnormally short TL, at or below the first percentile, and carried mutations in TERT $(n=5), T R(n=$ $6)$, or DKC1 $(n=3)$ or had familial forms of dyskeratosis congenita $(n=2)$ (Supplemental Table 2). The 3-way comparison would allow us to test the contribution of short telomeres alone relative to the $\mathrm{T}$ cell changes that occur with aging. We first examined the distribution of peripheral T cells from each of the 3 groups to determine whether T cells may show the $\mathrm{T}$ cell-skewing pattern characteristic of the T cell-aging phenotype and found the ST group had markedly fewer naive $\left(\mathrm{CD} 45 \mathrm{RA}^{+} \mathrm{CCR} 7^{+}\right) \mathrm{CD}^{+}$and $\mathrm{CD} 8^{+} \mathrm{T}$ cells compared with age-matched controls (Figure 2, C-F). The extent of this decrease was similar to that in OA who were 50 years older. Since ST patients also had T cell lymphopenia (Figure $1 \mathrm{~F}$ ), this result indicated that the absolute naive $\mathrm{T}$ cell pool was extremely depleted in ST patients. Concurrently, and also similarly to OA, ST patients accumulated terminally differentiated $\mathrm{CD}^{+}$effector memory CD45RA ${ }^{+} \mathrm{T}$ cells (CD45RA ${ }^{+} \mathrm{CCR} 7^{-}$, TEMRA), which made up the majority of circulating $\mathrm{CD} 8^{+} \mathrm{T}$ cells (Figure 2, $\mathrm{E}$ and F). These data suggested that short telomeres are sufficient to drive the characteristic $T$ cell skewing that occurs with aging.

Telomerase mutation carriers have decreased thymic output. To understand why naive $\mathrm{T}$ cells are depleted in ST patients, we first examined thymic output by quantifying the proportion of circulating recent thymic emigrants (RTEs) $\left(\mathrm{CD} 4^{+} \mathrm{CD} 45 \mathrm{RA}^{+} \mathrm{CD} 31^{+}\right)$ (19) and found their fraction was somewhat decreased (Figure $2 \mathrm{G})$. This led us to directly test thymic function by quantifying circulating TCR excision circles (TRECs). This PCR-based assay is the current standard screening test across the United States and elsewhere for severe primary immunodeficiencies in newborns, with the goal of implementing timely treatment prior to the onset of fatal infections (20-22). Among the 10 ST patients we tested, $6(60 \%)$ had abnormally low TRECs below the age-adjusted fifth percentile $\left(P<0.001, \chi^{2}\right.$ test) (Figure $\left.2 \mathrm{H}\right)$. One of these cases was an infant who presented prior to implementation of newborn TREC screening and who had severe enterocolitis as a first presentation, similar to what has been described (16). He was found to carry a de novo DKC1 mutation (Figure $2 \mathrm{H}$ and Supplemental Table 2). His TREC levels were tested at the time of his diagnosis, at 8 months of age, and were found to be undetectable (Figure $2 \mathrm{H})$. These observations collectively indicated that some telomerase mutation carriers may be identified during TREC newborn-screening programs; their identification has implications for therapy, given the exquisite sensitivity of short telomere syndrome patients to standard therapies.

Mice with short telomeres show $T$ cell-autonomous apoptotic defects. To define how short telomeres may cause thymic export failure, we studied telomerase RNA null, $m \mathrm{TR}^{-/}$, generation 4 $\left(m T R^{-/-} G 4\right)$ mice, which acquire short telomeres after successive generations of breeding (23). We first tested whether short telomeres disturbed the thymic niche in mice and compared the capacity of the short telomere niche to support WT T cell development. We transferred WT hematopoietic stem-progenitor cells into WT or $m T R^{-/-} G 4$ recipients after a small sublethal dose of radiation (ref. 24 and experimental design in Figure 3A). The choice of this low-dose protocol was intentional, given the known radiosensitivity of short telomere mice and to minimize a skewed engraftment (25). We found bone marrow engraftment was similar in both 
Table 1. Characteristics of patients enrolled in the Johns Hopkins Telomere Syndrome Registry who developed opportunistic infections, their mutation, and bone marrow function

$\begin{array}{lcc}\text { Patient } & \text { Age (yr) } & \text { Sex } \\ 1 & 0 & \mathrm{M} \\ 2 & 1 & \mathrm{~F} \\ 3 & 18 & \mathrm{~F} \\ 4 & 19 & \mathrm{~F} \\ 5 & 23 & \mathrm{M} \\ 6 & 33 & \mathrm{M} \\ 7 & 36 & \mathrm{~F} \\ 8 & 48 & \mathrm{~F} \\ 9 & 61 & \mathrm{M}\end{array}$

Sex
M
F
F
F
M
M
F
F
M

Cene/Mutation
DKC1, A308C
RTEL1, R1264H;:R1264H
TERT, K902N
TERT, V1025F
TERT, C1350
DKC1, IVS2-5C>C
TR, del375-377
TR, G257T
TERT, L841F

\begin{tabular}{lcl}
\multicolumn{1}{c}{ Complication } & CD4 $(/ \mu \mathrm{l})^{\mathrm{A}}$ & Blood counts \\
\hline Enterocolitis & 621 & Normal \\
Enterocolitis & 753 & Normal \\
VZV reactivation & 442 & Normal \\
CMV encephalitis & 50 & Severe AA \\
VZV reactivation, Tinea cruris & 94 & Moderate AA \\
HPV-associated anal carcinoma & 195 & Mild AA \\
VZV reactivation & 399 & Normal \\
CMV pneumonia, P. jiroveci pneumonia & 386 & Severe AA \\
CMV pneumonia & 349 & Normal
\end{tabular}

${ }^{A}$ All CD4 counts noted are below the age-adjusted normal range. Absolute CD8 counts were available for patients 1, 3, 5, 7, and 8, and they fell near or below the age-adjusted range at 146, 187, 235, 256 and 185/ $\mu$, respectively. Patient 9 developed fatal CMV pneumonia after lung transplantation.

A

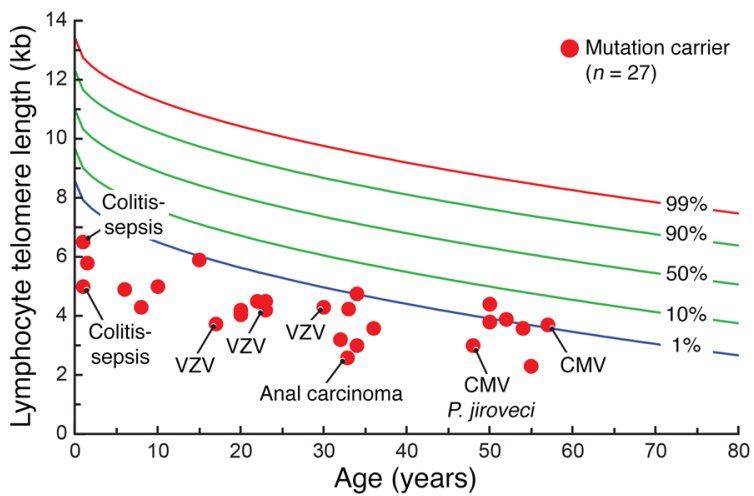

B

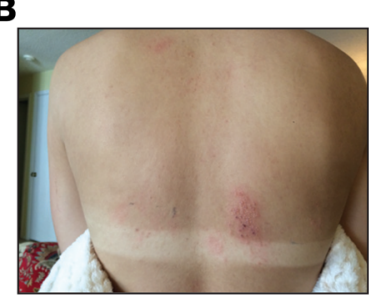

C

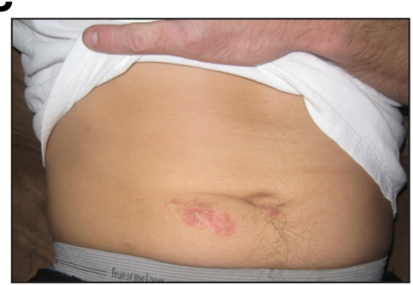

D

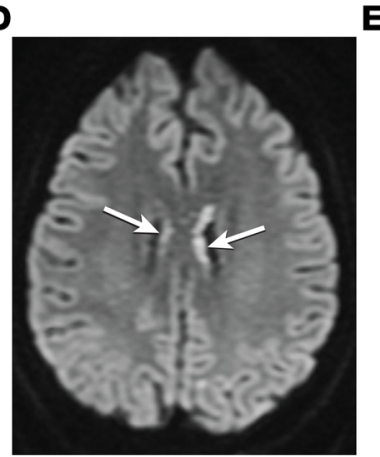

$\mathbf{F}$
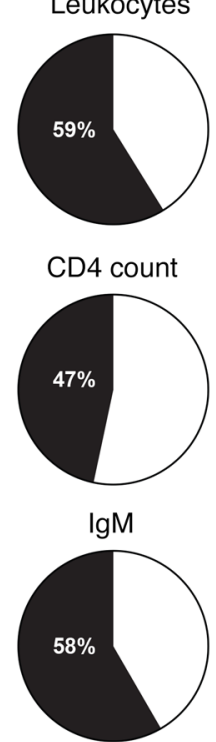

Absolute

lymphocyte count
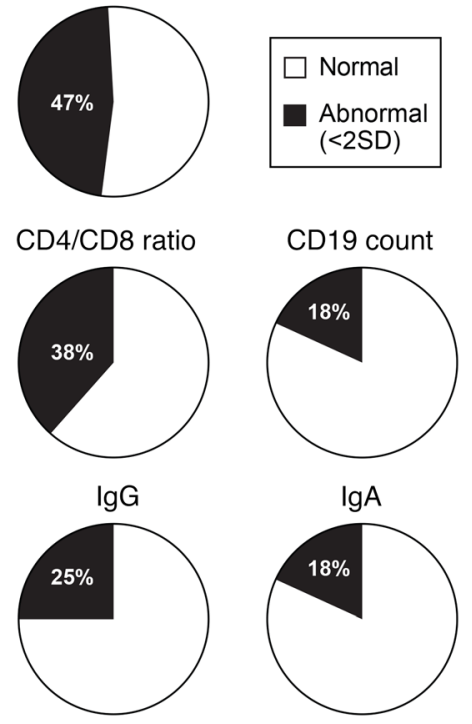

Figure 1. T cell primary immunodeficiency and its complications in telomerase mutation carriers. (A) Telogram showing total lymphocyte TL measured by flow cytometry and FISH of mutation carriers relative to a nomogram of healthy controls. Those who developed opportunistic infections are noted. One TERT mutation carrier (patient 4, Table 1) did not have TL measured, so only 27 of 28 patients studied are plotted. (B and C) Images showing vesicular rash characteristic of VZV reaction (patients 3 and 5 in Table 1, respectively). (D) Brain MRI showing evidence of enhancing periventricular flare (marked by arrows) in a 19-year-old who died from fatal CMV encephalitis (Table 1, patient 4). (E) Chest CT scan image from a patient who developed concurrent $P$. jiroveci pneumonia that was complicated secondarily by CMV pneumonitis; the latter was treatment refractory and ultimately fatal. (F) Proportion of telomerase mutation carriers with lymphocyte count abnormalities (defined as at least 2 SD below the age-adjusted mean). Low CD4 counts and low IgM levels were the most common anomalies. Data are derived from 17 patients, including 7 from Table 1 for whom the full immune evaluation was available. 
A
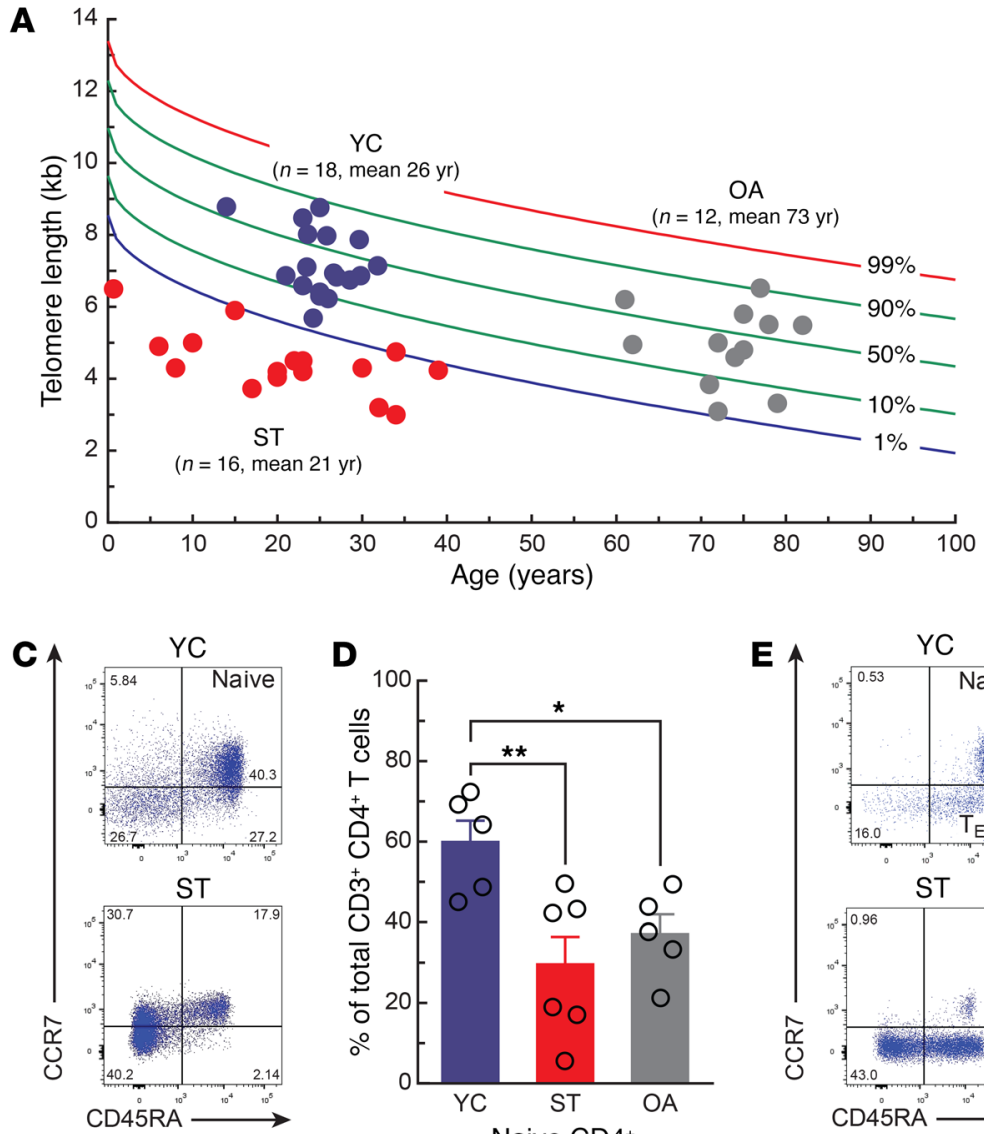

D
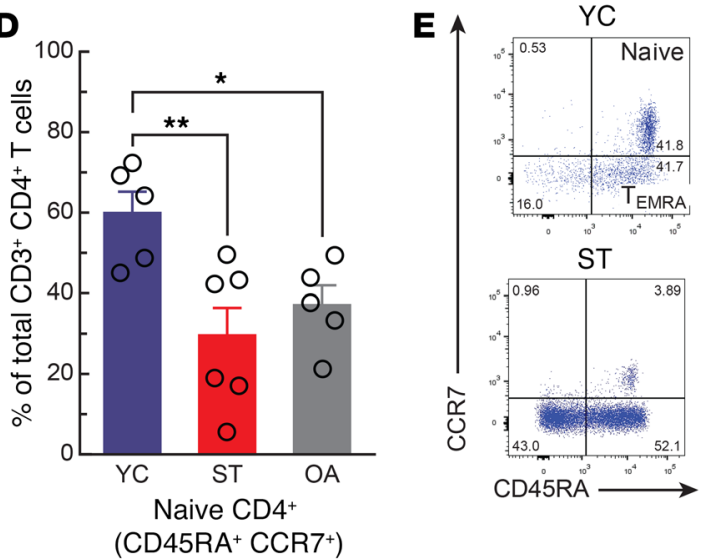

B

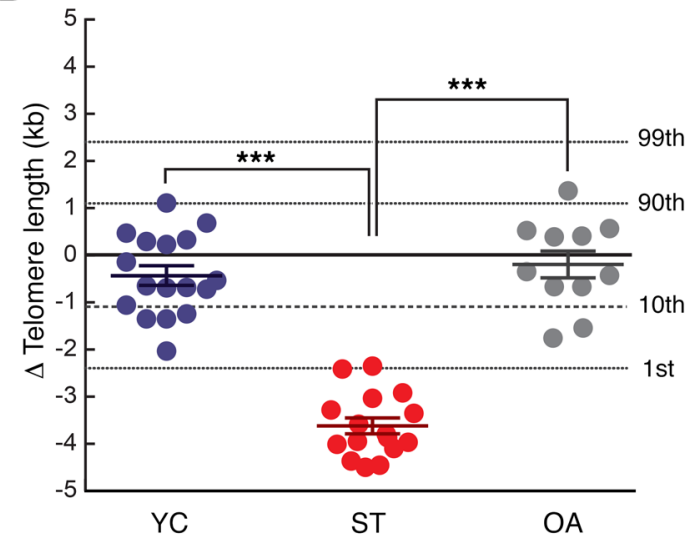

$\mathbf{F}$

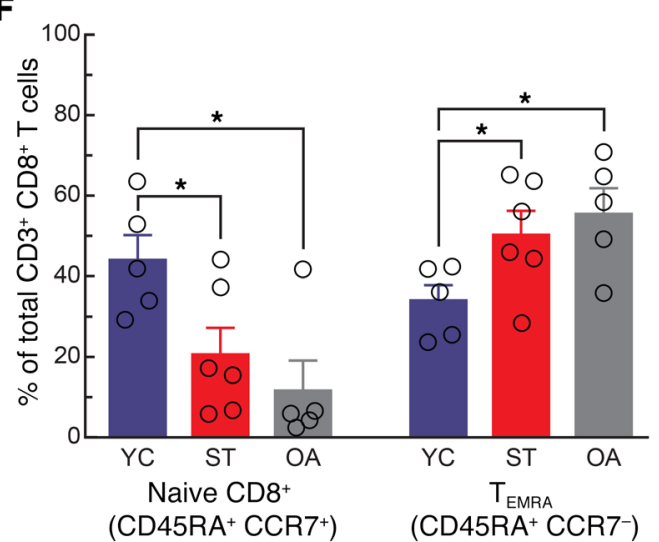

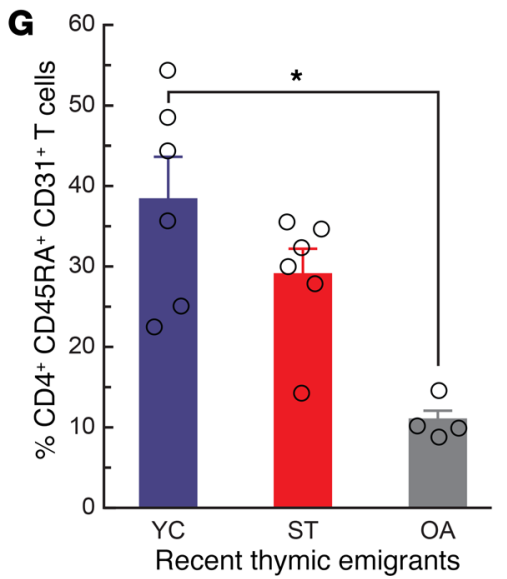

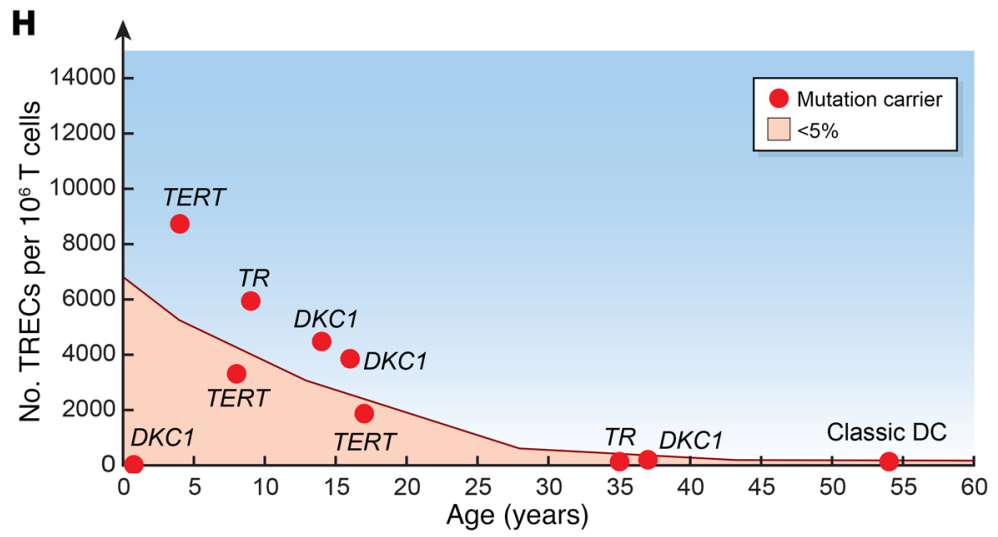

Figure 2. Telomerase mutation carriers have premature skewing of T cell subsets and decreased TRECs. (A) Telogram showing the age-adjusted lymphocyte TL for each individual falling in 1 of 3 groups studied. (B) Difference in TL from the age-adjusted median for cases shown in A. YC and OA groups cluster around the age-adjusted median, whereas ST patients are at or below the first percentile. (C) Representative flow plots of peripheral CD4 ${ }^{+}$T cells from $Y C$ and ST subjects. (D) Percentage of naive CD4 ${ }^{+} T$ cells, defined as $C D 3^{+} C D 4^{+} C D 45 R A^{+} C C R 7^{+}$. (E) Representative flow plots from $Y C$ and $S T$ cases showing

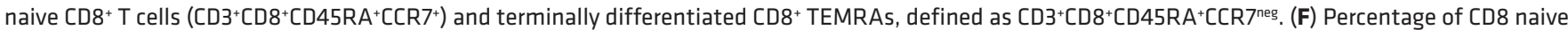
and TEMRA populations as defined in E. For C-F, $n=5$ YC, 2 male/3 female; $n=6 \mathrm{ST}, 2$ male/4 female; and $n=5$ OA, 3 male/2 female. (C) Quantification of RTEs defined as CD4+CD45RA+CD31+. $n=6 \mathrm{YC}, 2$ male/4 female; $n=6 \mathrm{ST}$, 3 male/3 female; $n=4 \mathrm{OA}, 2$ male $/ 2$ female. (H) TRECs measured by quantitative PCR in telomerase mutation carriers. Data from each of the 10 patients ( 5 male/ 5 female) are graphed relative to an age-adjusted nomogram with the fifth percentile shown. The normal range was derived from 254 controls. For 9 patients, the mutated gene is shown, and 1 patient had classic features of dyskeratosis congenita (DC). In an infant with DKC1 mutation, TREC levels were undetectable. Error bars represent SEM. ${ }^{*} P<0.05$; ${ }^{* *} P<0.01$; ${ }^{* *} P<$ 0.001 , Student's $t$ test, 2 -sided. 
A bone marrow HSPCs

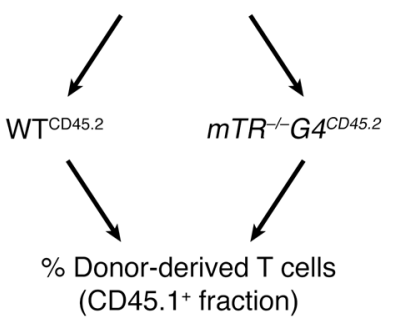

D

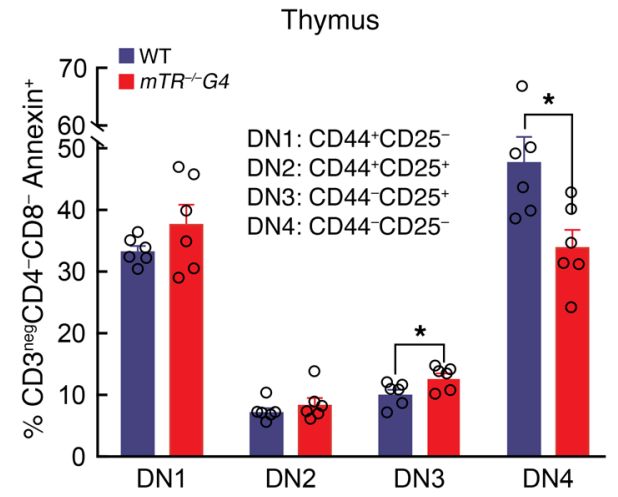

H

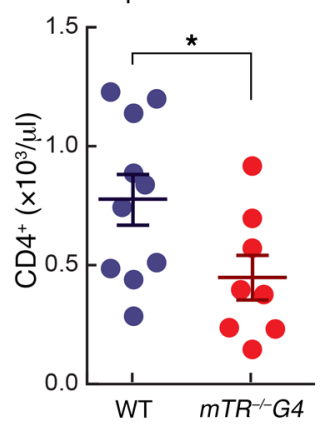

B

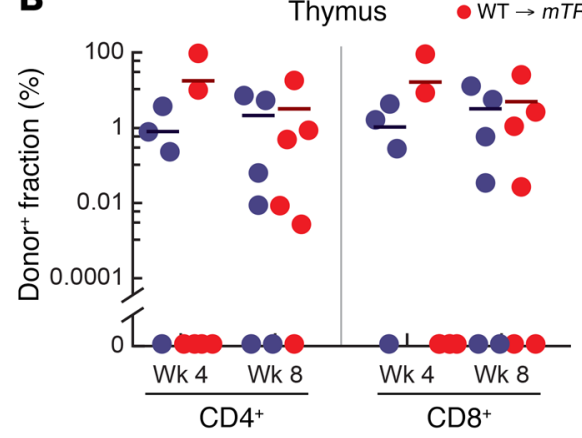

C Peripheral blood $\begin{aligned} & \bullet W T \rightarrow W T \\ & \bullet W T \rightarrow m T R^{-}-G 4\end{aligned}$

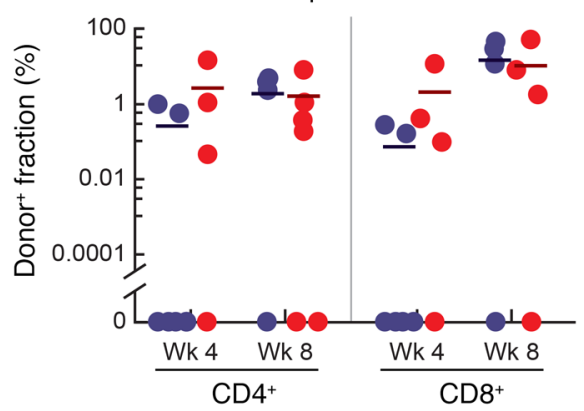

E Thymus

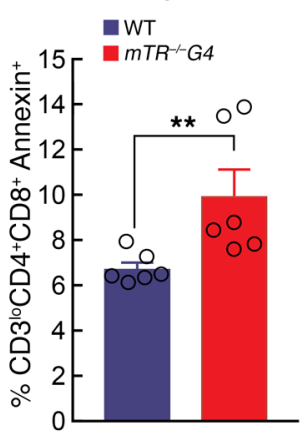

J Peripheral blood

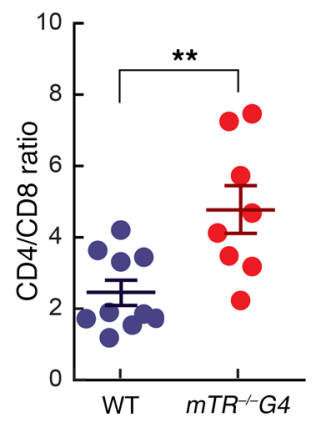

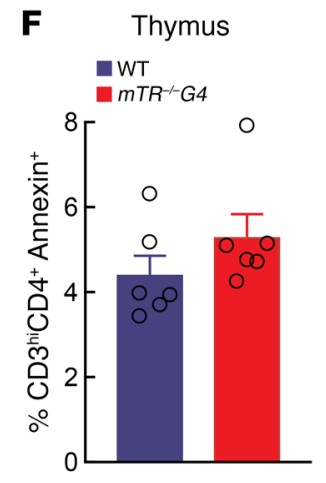

G Thymus

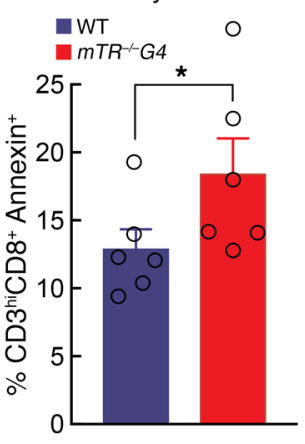

K Peripheral T cells

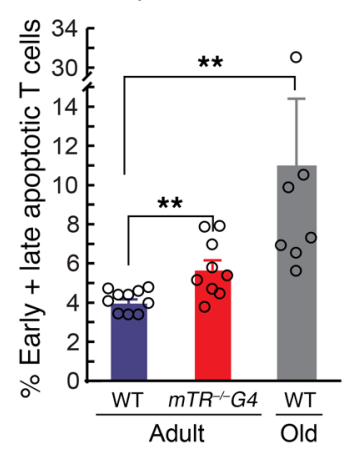

L Human peripheral

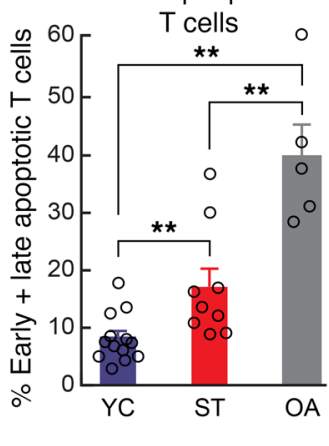

Figure 3. T cell-intrinsic apoptosis contributes to T cell lymphopenia. (A) Schematic for congenic transplant of bone marrow hematopoietic stem progen-

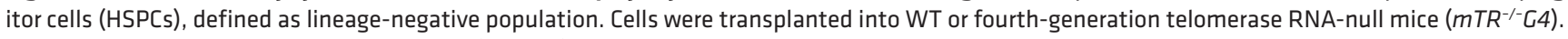
Donor-derived T cell fractions were assessed. (B and C) Quantification of donor-derived CD4 ${ }^{+}$and CD8 ${ }^{+}$T cells in thymuses and peripheral blood at 4 and 8 weeks after transplantation for WT and $m T R^{-1-} G 4$ recipient mice ( $n=6$ recipients were studied, 3 male/3 female for each genotype at each time point). (D) Thymocyte apoptosis rates in $\mathrm{CD}^{\text {nes }} \mathrm{CD} 4^{-} \mathrm{CD} 8^{-}(\mathrm{DN})$ populations 1, 2, 3, and 4, defined by their cell-surface markers, as shown. (E) Apoptotic fraction of $\mathrm{CD}^{\text {lo }} \mathrm{CD}^{+} \mathrm{CD} 8^{+}$(DP) thymocytes in newborn. (F and $\left.\mathbf{G}\right)$ Apoptotic fraction of $\mathrm{CD} 3^{\text {hi }} \mathrm{CD} 4^{+}$and $\mathrm{CD} 3^{\text {hic }} \mathrm{CD} 8^{+}$thymocytes. For $\mathbf{D}-\mathbf{G}$, the apoptotic fraction was quantified as the total annexin $\mathrm{V}^{+}$population in newborn mice (1 week old, $n=6 /$ group, sex not determined because of young age). (H-J) Peripheral blood absolute $\mathrm{CD}^{+}, \mathrm{CD}^{+} \mathrm{T}$ cell counts and the CD4/CD8 ratio, respectively. For H-J, $n=10 \mathrm{WT}, 7$ male/3 female, $n=9 \mathrm{mTR} /-\mathrm{C4}, 4$ male $/ 5$ female, $6-16$ weeks). (K) Peripheral T cell apoptosis quantified at 48 hours as total annexin $\mathrm{V}^{+} \mathrm{P}^{\text {nes }}$ plus annexin $\mathrm{V}^{+} \mathrm{Pl}^{\text {lo }}$ populations for splenocytes stimulated with $\mathrm{CD} 3 / \mathrm{CD} 28$. Total T cells were isolated from 10 WT (7 female/3 male) and 9 mTR ${ }^{-/-}$- 4 (4 female/3 male), 20-25 weeks; 7 old WT (5 female/2 male), 55-67 weeks. (L) Apoptosis quantified at 48 hours (as in K) for T cells isolated from peripheral blood of YC ( $n=13,8$ male/5 female), ST ( $n=9$, 3 male/6 female), and OA $\left(n=5,3\right.$ male/ 2 female). The analysis for $\mathbf{K}$ and $\mathbf{L}$ included total T cells. Error bars represent SEM. ${ }^{*} P<0.05$; ${ }^{* *} P<0.01$, Mann-Whitney $U$ test.

groups of recipients (Supplemental Figure 1A). While overall T cell engraftment was lower, given the small dose of radiation utilized, $\mathrm{CD}^{+}$and $\mathrm{CD}^{+} \mathrm{T}$ cell fractions in the thymus and periphery were similar in WT and $m \mathrm{TR}^{-/-} \mathrm{G} 4$ recipient mice (Figure 3, B and C). These data indicate that the thymic niche of short telomere mice can support normal thymocyte development and export.

Next we determined whether short telomeres compromise $\mathrm{T}$ cell development. We first examined hematopoietic stem-progen- itor precursors quantified by c-Kit ${ }^{+} \mathrm{Sca}^{-} \mathrm{Lin}^{-}(\mathrm{KSL})$ and $\mathrm{CD} 150^{+} 48^{-}$ Lin $^{-} \mathrm{C}-\mathrm{Kit}^{+}$(SLAM) markers (Supplemental Figure 1, B-E) and found they were severely depleted in short telomere mice, as has been seen previously (26). Hematopoietic progenitors from short telomere mice also had a severe engraftment defect when transplanted into WT mice (Supplemental Figure 1, F and G), similar to what had been seen previously (26). These data are consistent with the known repopulation defects of short telomere cells and 
indicate that an autonomous hematopoietic stem-progenitor defect at least partially contributes to $\mathrm{T}$ cell deficiencies caused by short telomeres.

We next tested whether there were additional thymocyte developmental defects that compound those caused by hematopoietic stem-progenitor failure. When we examined thymocyte numbers, we found them to be generally intact in both newborn and adult short telomere mice (Supplemental Figure 2, A and B). We then specifically examined $\mathrm{T}$ cell precursors marked by a double-negative population, CD3-CD4-CD8 ${ }^{-}$(DN). Using CD44 and CD25 antibodies to distinguish the 4 developmental DN populations (ref. 27 and Supplemental Figure 2C), we found trends toward increased rates of apoptosis in DN1, DN2, and DN3 populations, although this was only statistically significant for DN3 (Figure 3D). A similar pattern could be seen in adult mice (Supplemental Figure 2D); while these trends were not statistically significant, they suggested $\mathrm{T}$ cell attrition occurs during intrathymic $\mathrm{T}$ cell development during development and in adult mice. We noted that, for the DN4 population, in both newborn and adult mice, the trend was reversed, with increased apoptosis in WT mice (Figure 3D and Supplemental Figure 2D); the reasons for this pattern are unclear. However, in several of the remaining $\mathrm{T}$ cell developmental stages, we found relatively increased apoptosis, including in $\mathrm{CD} 4^{+} \mathrm{CD} 8^{+}$ double-positive (DP) and committed single-positive (SP) thymocytes with short telomere CD8 ${ }^{+}$SP cells having the statistically significant and highest rates in both newborns and adults (Figure 3, E-G, and Supplemental Figure 2, E-G, respectively). The cumulative attrition was most evident when we quantified peripheral $\mathrm{T}$ cells, which showed severe depletion in $m \mathrm{TR}^{-/-} \mathrm{G} 4$ mice. They had only $57 \%$ and $36 \%$ of WT absolute $\mathrm{CD}^{+}$and $\mathrm{CD} 8^{+} \mathrm{T}$ cell counts, respectively (Figure 3, $\mathrm{H}^{-} \mathrm{J}$, and Supplemental Figure 3, A-F). This magnitude of lymphopenia mirrors the defects we documented in the ST patients we studied (Table 1 and Figure 1).

To definitively test whether the $\mathrm{T}$ survival defects were cell autonomous, we studied the responses of $\mathrm{CD}^{+} \mathrm{T}$ cells ex vivo after stimulation with anti-CD3 and anti-CD28 antibodies. We first tested whether there were any cell-cycle defects by incubating the stimulated T cells with EdU, but we found no differences in the cell-cycle profiles (Supplemental Figure 4A). In contrast, when we examined annexin $\mathrm{V}$ expression, we found that stimulated $m T R^{-/-G 4-d e r i v e d ~} \mathrm{~T}$ cells showed higher rates of apoptosis, nearly $30 \%$ higher (Figure 3K and Supplemental Figure 4B). The significant increase in apoptosis was similar to that in aged WT mice, which are known to have normal TL (Figure 3K and Supplemental Figure 4B). To test the relevance of this finding to the patients we studied, we purified total $\mathrm{CD}^{+} \mathrm{T}$ cells and stimulated them similarly ex vivo. We again found no change in proliferation rate measured by EdU incorporation; however, similarly to those in short telomere mice, T cells from ST patients showed increased apoptosis relative to $\mathrm{YC}$, comparable to that in OA (Figure 3L and Supplemental Figure 4, C and D). Together, these data from ST patients and mice indicated that short telomere $\mathrm{T}$ cells have autonomous defects that predispose them to apoptosis similarly to what is seen in aged WT mice and OA humans.

TCR diversity is restricted in telomerase mutation carriers. The increased apoptosis we observed led us to ask whether higher rates of apoptosis in vivo over a lifetime may predispose ST patients to have a restricted TCR diversity, which would further compound their quantitative immunodeficiency. To test this hypothesis, we first examined the TCR repertoire by flow cytometry, quantifying the frequency of $\mathrm{T}$ cells expressing each of $24 \mathrm{TCR}-\mathrm{V} \beta$ proteins for which expression in controls is defined based on data from 85 healthy individuals (28). These V $\beta$ proteins represent approximately $70 \%$ of the TCR repertoire. For each individual, and across the 3 study groups, we compared the expression of each of the 24 TCR-V $\beta$ families relative to the control range, quantifying the number of TCR-V $\beta$ families that were 1 or 2 SD from the mean (example in Supplemental Figure 5A and summarized in Figure 4A). YC had minimal deviations from healthy controls, as expected, but ST patients had a significant divergence in total $\mathrm{CD}^{+}$and $\mathrm{CD}^{+} \mathrm{T}$ cells. The extent of this divergence in ST individuals was similar to that in OA (Figure 4, A-D). The greatest divergence was in $\mathrm{CD}^{+} \mathrm{T}$ cells, where half (12 of 24 ) of the TCR-V $\beta$ families analyzed were over- or underrepresented by at least $1 \mathrm{SD}(P=0.02$ for comparison to YC, Mann-Whitney $U$ test, Figure 4D).

To further assess the mechanisms underlying the restricted $\mathrm{CD}^{+}$TCR repertoire, we performed an unbiased, high-resolution sequencing analysis of the highly variable complementaritydetermining region 3 (CDR3) using immunoSEQ (Adaptive Biotechnologies) (29). We compared YC and ST individuals and found that the distribution of CDR3 lengths, as well as the addition of nucleotides by TdT at V-D and D-J junctions, was similar (Supplemental Figure 5B). This result indicated that the loss of diversity occurred after effective recombination and TCR assembly. In this immunoSEQ analysis, we also found evidence for a restricted TCR repertoire. ST cases had on average $18 \%$ fewer unique CDR3 sequences and a $13 \%$ reduction in the relative richness, defined as the average number of unique rearrangements per $\mathrm{CD} 8^{+} \mathrm{T}$ cell sequenced (Figure 4, E and F). The restricted diversity was independently reflected in a lower mean Pielou's $\mathrm{J}$ index of $\mathrm{V} \beta$ gene usage, a calculation of the uniformity, where 0 is complete redundancy and 1 is perfect evenness (30) (Figure 4G). We also found evidence for an asymmetric loss of diversity. The usage of the 5 most frequently represented $\mathrm{V} \beta$ families, the most highly utilized, was overrepresented in the ST group (Figure $4 \mathrm{H}$ ). Conversely, the next 15 frequently utilized $\mathrm{V} \beta$ genes were underrepresented (Figure $4 \mathrm{H}$ ). Similar trends in $V \beta$ usage have been documented in $\mathrm{OA}$ $\mathrm{T}$ cells (31). The skewing of the repertoire toward greater usage of the more common $\mathrm{V} \beta$ genes and lower use of the less common genes is consistent with sampling a population that has undergone a drastic reduction in size and subsequent loss of diversity.

The short telomere-associated apoptosis program is distinct from that in OA with normal TL. Our data so far supported that short telomere syndrome patients have a $\mathrm{T}$ cell immunodeficiency that is both quantitative and qualitative and that it is driven in part by $\mathrm{T}$ cell apoptosis. To begin to understand the mechanisms underlying this apoptotic defect, we examined the gene expression pattern of CD8 ${ }^{+}$TEMRAs (schematic in Supplemental Figure 6). We chose this population because it was the most abundant in ST patients and OA, constituting more than half of circulating T cells (Figure $2 \mathrm{~F}$ ). We found that the extent of intergroup variance in gene expression was greater among the 3 groups than within each of them (Figure 5A). Moreover, unexpectedly, there was no major overlap in the expression pattern of genes in the ST group with 
A

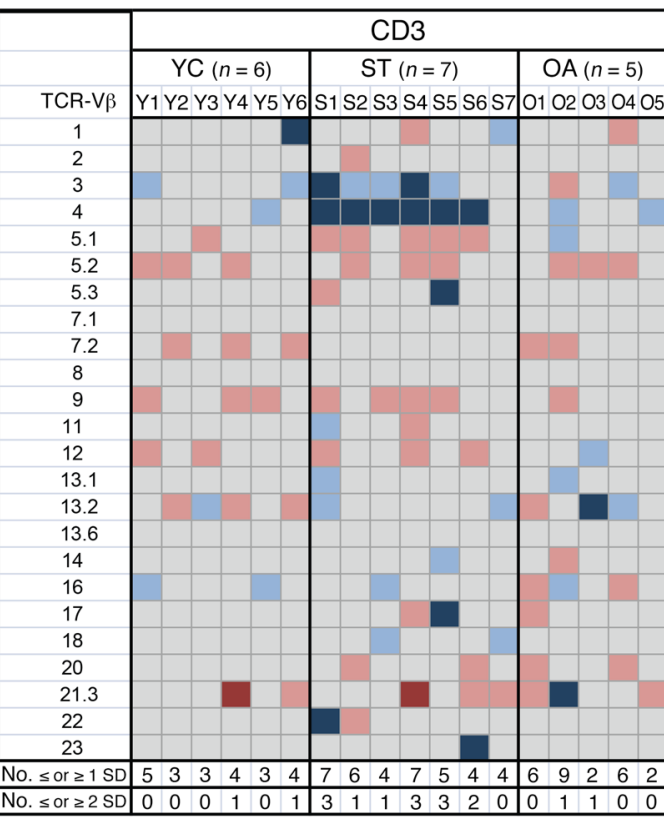

$\square-1$ to -2 SD $\quad \square \pm 0-1$ SD $\quad \square 1$ to 2 SD $\quad \square \geq 2$ SD

B

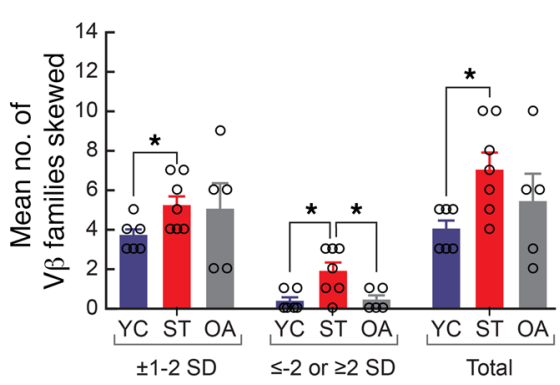

E

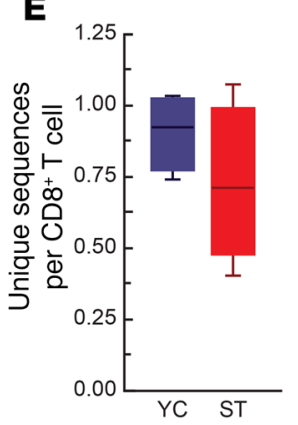

$\mathbf{F}$

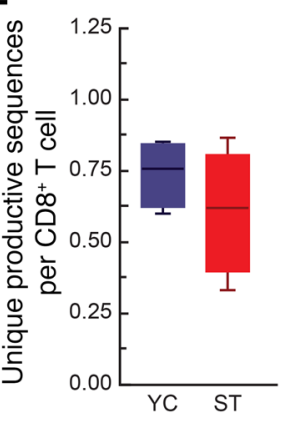

C

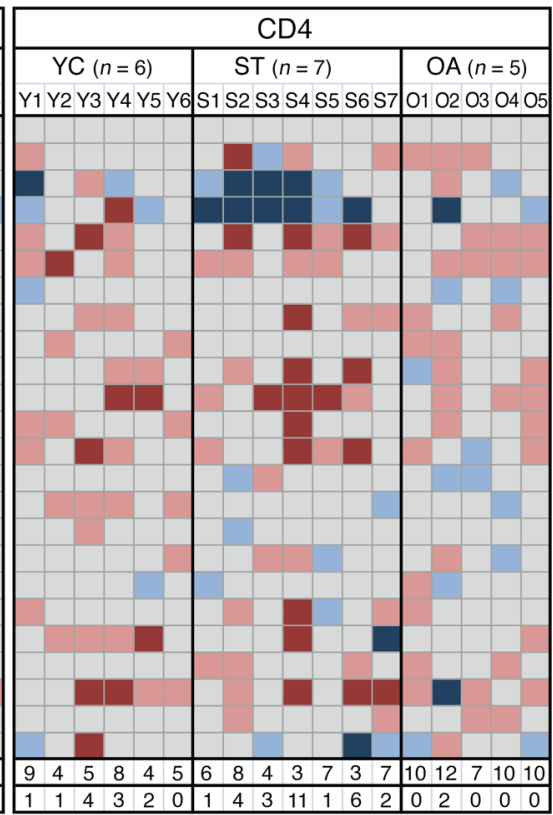

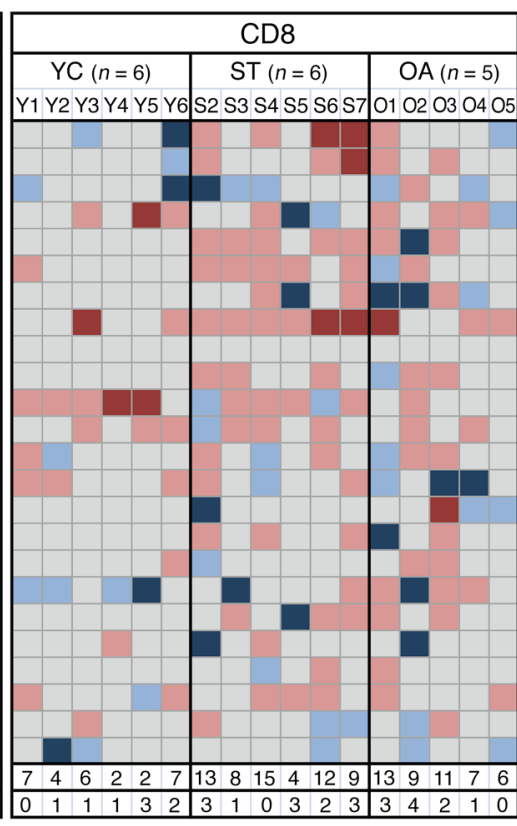

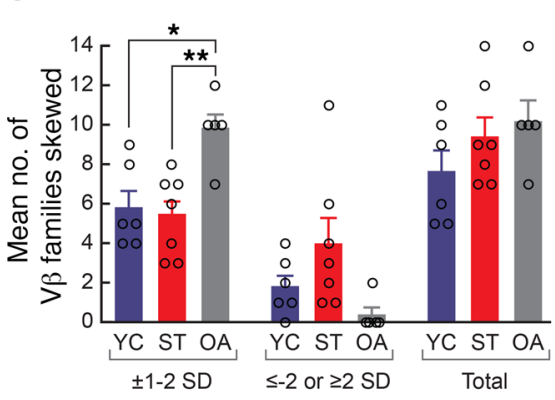

D

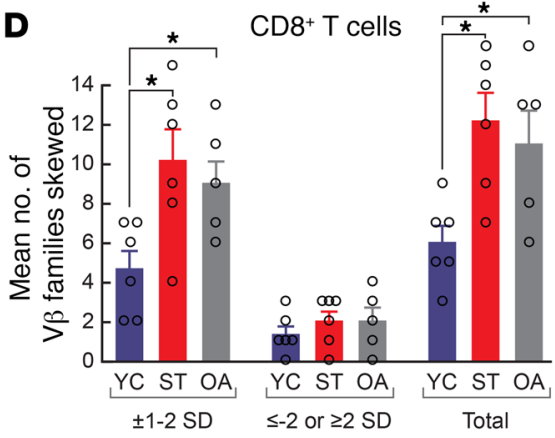

G

Pielou's index ( $\mathrm{J}$ ) $\begin{array}{lllllllll}0.80 & 0.81 & 0.82 & 0.83 & 0.84 & 0.85\end{array}$

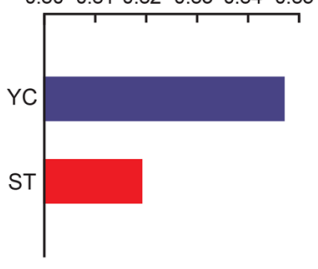

H

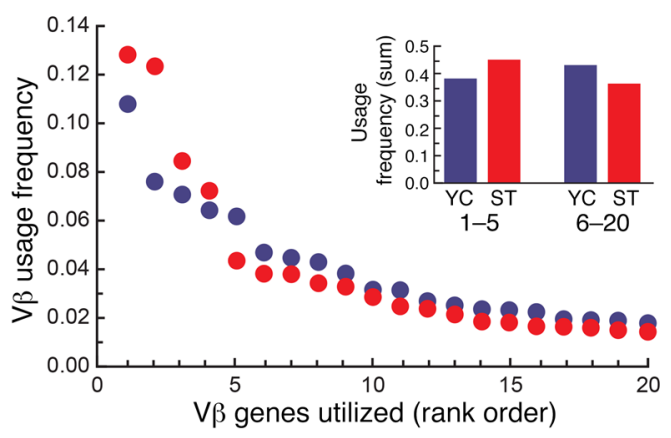

Figure 4. The TCR repertoire is restricted in telomerase mutation carriers. (A) Expression of $24 \mathrm{~V} \beta$ proteins by flow cytometry for YC, ST, and OA relative to 85 controls. Each column represents data from a single individual, with gray representing percentage of V $\beta$-expressing T cells within $1 \mathrm{SD}$ from the mean and the colors representing greater deviation from means derived from controls. The degree of deviation is noted in the key. Data are shown for $\mathrm{CD}^{+}$, $\mathrm{CD}^{+}$, and CD8 ${ }^{+} T$ cells and are summarized in the bottom 2 rows. (B-D) Bar graphs show mean number of $V \beta$ families deviating 1-2, or more than 2, SD for CD3', $\mathrm{CD}^{+}$, and CD8 ${ }^{+}$T cells, respectively. $n=6$ YC, 2 male $/ 4$ female; $n=7 \mathrm{ST}, 2$ male $/ 5$ female; $n=5 \mathrm{OA}, 3$ male $/ 2$ female. Error bars in B-D represent SEM. (E) T cell diversity as measured by the mean unique sequences per T cell determined by deep sequencing of the CDR3 of the TCR- $\beta$ gene on sorted CD8 ${ }^{+}$cells ( $n$ = 4/group, 2 male/2 female for each). (F) Unique productive sequences per CD8 ${ }^{+} T$ cell for data generated for $\mathbf{E}$. Whiskers in $\mathbf{E}$ and $\mathbf{F}$ mark the minimum and maximum values. (C) Pielou's J index, a calculation of evenness of $V \beta$ usage where 1 represents an even distribution and 0 represents complete dominance of $1 \mathrm{~V} \beta$. (H) Dot plot of total usage of the 20 most frequently utilized V $\beta$ genes in YC compared with ST subjects shows a higher usage in ST patients. Inset shows summed usage frequency of the top 5 most frequently used $V \beta$ genes; the increased usage of highly utilized genes is reflected in a decreased usage of the subsequent $\mathrm{V} \beta$ genes (i.e., those ranked 6 to 20 ). ${ }^{*} P<0.05$; ${ }^{* *} P<0.01$, Mann-Whitney $U$ test. 
A

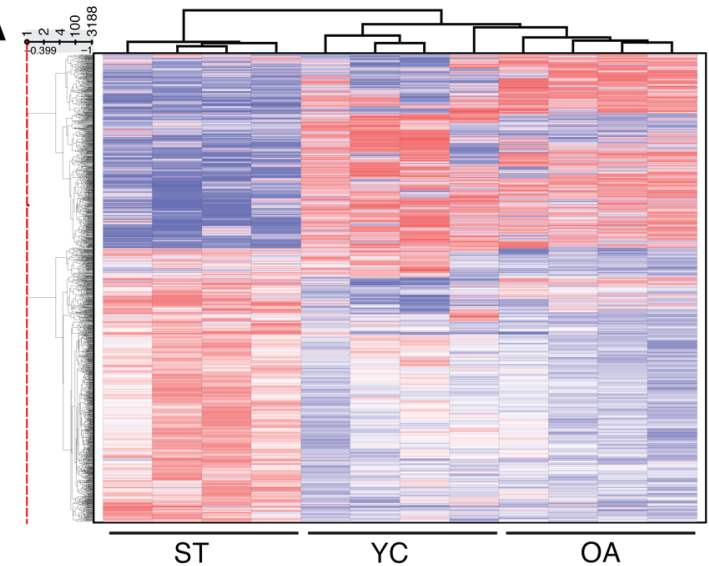

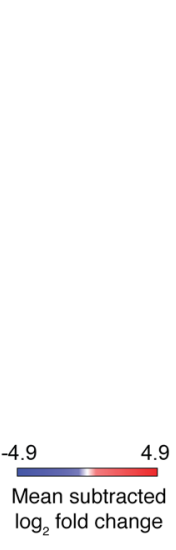

B

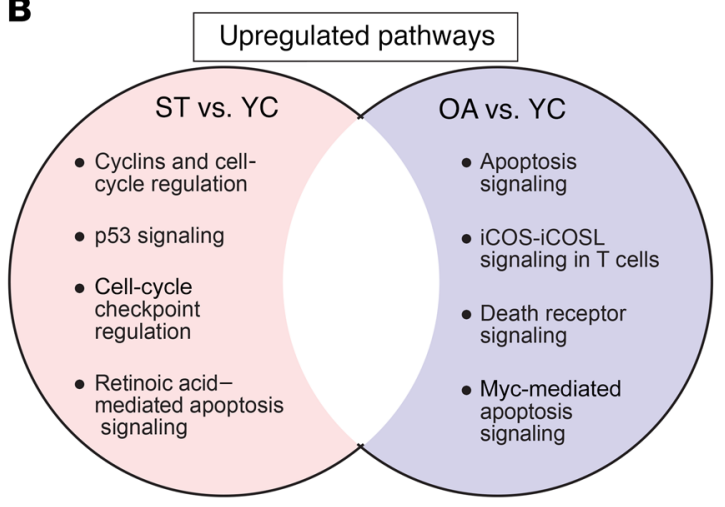

C

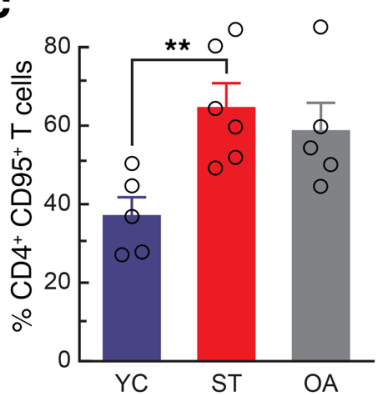

D

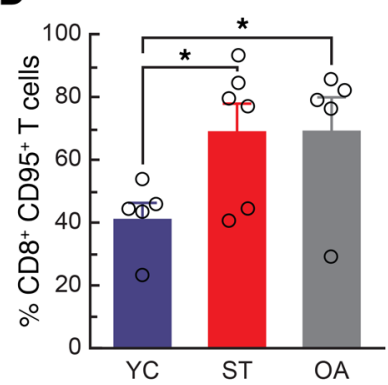

E

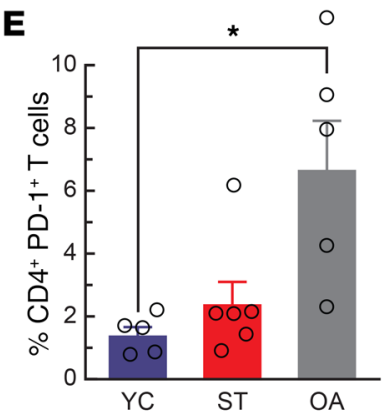

$\mathbf{F}$

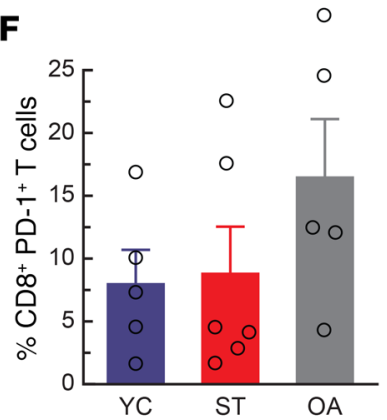

G

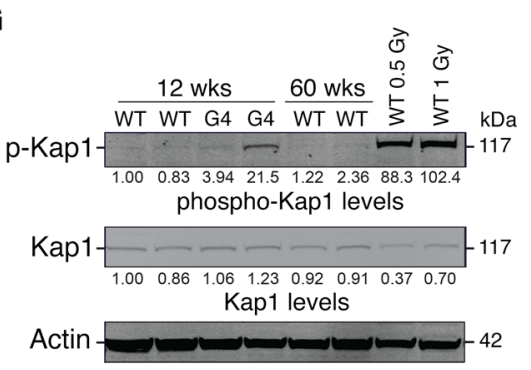

J

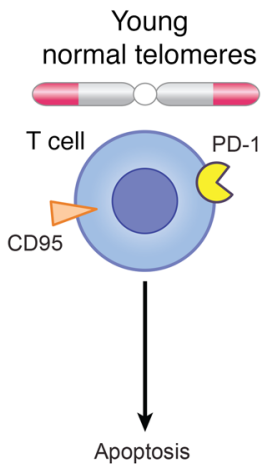

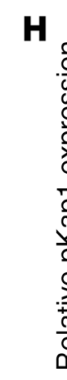

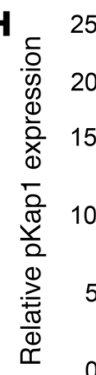

Young short telomeres

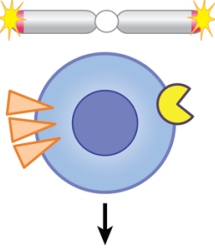

DNA damage response

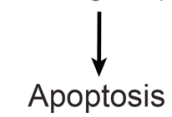

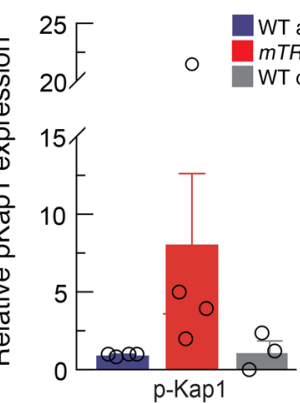

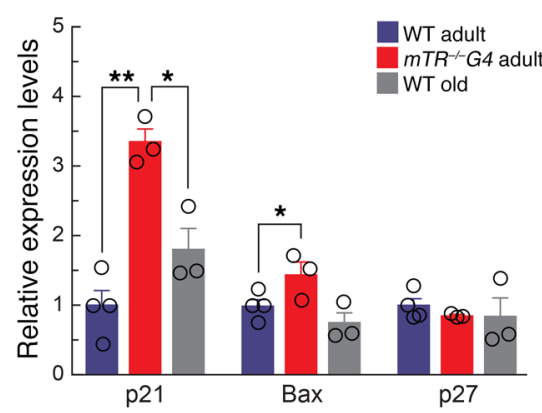

Aged normal telomeres

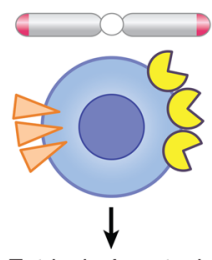

Extrinsic Apoptosis

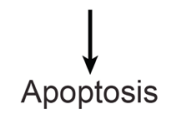

Aged

short telomeres

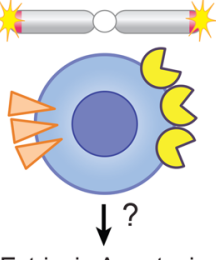

Extrinsic Apoptosis DNA Damage Response

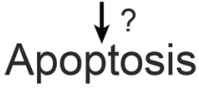

Figure 5. Distinct pathways to apoptosis in short telomere and OA T cells. (A) Heatmap and dendrogram of gene expression showing the mean subtracted expression values on a $\log _{2}$ scale. For each of 12 samples, YC, ST, and OA groups ( 2 male/2 female/group), the log 2 expression value was subtracted from the mean $\log _{2}$ expression value of the entire cohort. The dendrogram showing relatedness of the samples is above, and relatedness of the gene transcripts is to the left. The differential change in gene expression is shown as positive and negative change on color scale indicated in key. (B) Venn diagram shows 4 of 20 nonoverlapping upregulated pathways in IPA involved in apoptosis. (C and D) CD95 expression in CD4+ and CD8 ${ }^{+}$T cells, respectively. (E and F) PD-1 expression in $\mathrm{CD}^{+}$and $\mathrm{CD} 8^{+}$T cells, respectively. For C-F, $n=5 \mathrm{YC}, 2$ male/3 female; $n=6$ ST, 2 male/4 female; and $n=5 \mathrm{OA}, 3$ male/2 female. (G and $\mathbf{H}$ ) Kap1 and p-Kap1 levels on protein from isolated mouse T splenocytes. p-Kap1 and actin were detected first. Then the blot was stripped and reblotted with Kap1 antibody. Protein from irradiated splenocytes is a positive control. (H) Shown are quantification data from 3 independent Western blots from a total of 11 mice: WT (30 weeks, 1 male/3 female), $m T^{-1-} \mathrm{C} 4$ (30-33 weeks, 4 female), and old WT mice (50-73 weeks, 3 female). (I) qRT-PCR from unstimulated T splenocytes. Each data point represents an independent experiment with ages similar to those in $\mathbf{H}$. (J) Model of T cell-aging mechanisms showing differences in immunophenotype and T cell apoptosis program in young ST T cells and OA with normal TL. Older individuals with short telomeres are predicted to have extrinsic and intrinsic apoptotic mechanisms contributing. Error bars represent SEM. ${ }^{*} P<0.05$; ${ }^{*} P<0.01$, Student's $t$ test. 
those in OA or YCs (Figure 5A). In fact, YC and OA shared a far more similar gene expression pattern than ST (Figure 5A).

To test the functional relevance of this observation, we examined the most differentially expressed genes in ST and OA (2-fold difference) relative to YC using the Ingenuity Pathway Analysis (IPA) platform. Among the top 20 upregulated pathways, there was no overlap between the ST-YC and OA-YC comparisons (Supplemental Figure 7A), and only 3 of the top 20 downregulated pathways were shared (Supplemental Figure 7B). Nevertheless, among the upregulated pathways, we noted in both comparisons that there were pathways related to cell survival and that 4 pathways within each comparison group involved apoptosis (Figure 5B). In the ST versus YC comparison, these upregulated pathways included cell-cycle regulation, p53 signaling, checkpoint regulation, and retinoic acid-mediated apoptosis signaling (Figure $5 \mathrm{~B}$ ). In the OA versus YC comparison, the pathways included apoptosis signaling, iCOS signaling, death receptor signaling, and Myc-mediated apoptosis (Figure 5B). These analyses raised the possibility that divergent mechanisms, intrinsic apoptosis for ST T cells and extrinsic apoptosis in OA, converge phenotypically to contribute to the survival defects we documented (Figure $3 \mathrm{~L}$ ), but may be driven by distinct programs.

To determine whether ST and OA T cells may have distinct apoptotic signaling mechanisms, we analyzed their cell-surface markers. Both ST and OA showed upregulated CD95 expression, consistent with their apoptosis-prone state (Figure 5, C and D, and Supplemental Figure 8, A and B). In contrast, only OA T cells upregulated the expression of the programmed cell death 1 (PD-1) receptor (Figure 5, E and F, and Supplemental Figure 8, C and $\mathrm{D}$ ). There was also a trend for OA, but not ST T cells, to have a greater loss of $\mathrm{CD} 28$, the costimulatory molecule required for $\mathrm{T}$ cell activation, and to upregulate $\mathrm{CD} 57$ expression on $\mathrm{CD}^{+} \mathrm{T}$ cells (Supplemental Figure 8, E-H); both of these latter features have been associated with age-related immunophenotypes (32). These observations supported the possibility that distinct pathways may be contributing to ST and OA apoptosis and that extrinsic signaling is associated with OA T cells, but not those from ST patients.

It is established that short telomeres activate a DNA-damage response that is p53 dependent and that this response mediates the cellular short telomere phenotype (6). Since both mouse short telomere and old WT T cells are prone to apoptosis (Figure $3 \mathrm{~K}$ ), we tested to determine whether the DNA damage signal is restricted to $m T^{-1-} G 4 \mathrm{~T}$ cells. We thus isolated mouse $\mathrm{T}$ cells and examined them for evidence of a DNA double-strand break response. We first quantified baseline phosphorylated Kap1 (p-Kap1) protein levels, a downstream effector of ATM signaling and a known effector of short dysfunctional telomeres. We found that p-Kap1 levels, relative to total Kap1 levels, were upregulated in irradiated T cells, as would be expected (Figure 5, G and H; see also complete, unedited blots in the supplemental material). Importantly, T cells from $m T R^{--} G 4$ mice similarly had increased p-Kap1 levels, but old WT mice lacked this signal (Figure 5, G and H; see also complete, unedited blots in the supplemental material). We next tested the relevance of the gene expression microarray results implicating p53 signaling. To do so, we quantified the mRNA expression of p53 targets and found the levels of p21 and Bax were significantly increased in $m \mathrm{TR}^{-/-} \mathrm{G} 4$, but not in old WT T cells (Figure 5I). In contrast, the expression levels of p27, a p53-indepen- dent cyclin-dependent kinase inhibitor, were unchanged (Figure 5I). Thus, even though $\mathrm{T}$ cells with short telomeres share a propensity to apoptosis with old WT T cells (Figure 3K), they uniquely upregulate a p53-related, DNA double-strand break response.

\section{Discussion}

Although telomeres shorten with age in humans, their role in driving human aging and age-related disease is still incompletely understood. Here, we show that short telomeres are sufficient to mediate a T cell-aging phenotype, but only at abnormal thresholds. In individuals with germline mutations in telomerase, we identified a quantitative and qualitative $T$ cell immunodeficiency that causes severe and, at times, fatal complications. While abnormal immune indices have been reported in children with dyskeratosis congenita $(34,35)$, we found complications related to a telomere-mediated $\mathrm{T}$ cell immunodeficiency may be a first presentation in the absence of all the other stigmata of telomere-mediated disease, including bone marrow failure. In some cases, these individuals may be identified presymptomatically during TREC-based newborn screening. These observations add the disease-causing telomere and telomerase genes to the causes of primary immunodeficiency. Recognizing affected patients through genetic testing and clinical quality TL measurement is critical, since in contrast with most patients with primary immunodeficiency, these individuals are highly susceptible to fatal toxicities from conventional therapies, including myeloablative stem cell transplantation, and attenuated treatment protocols are necessary $(9,36,37)$.

While young telomerase mutation carriers had a 5-decade acceleration of $\mathrm{T}$ cell-aging phenotypes, the extent of their immunodeficiency was far greater than that of OA. This likely occurs because of ongoing and persistent depletion of precursors in the bone marrow, thymus, and periphery of individuals who are born with short telomeres. To our surprise, we also found that short telomere $\mathrm{T}$ cells had a distinct molecular signature. Short telomere patient-derived $\mathrm{T}$ cells also showed evidence of a DNA-damage double-strand break response and upregulated intrinsic apoptosis, in contrast with OA T cells with normal TL, which showed a gene expression profile consistent with extrinsic apoptosis that was associated with PD-1 upregulation. Thus, although short telomeres are acquired with aging, there appears to be a threshold where this shortening is biologically and clinically relevant and sufficient to signal telomere dysfunction on a cellular level. Such a threshold may not be reached in most individuals with aging. One or a few short telomeres are sufficient to induce cellular phenotypes, and the threshold of 400 base pairs has been suggested to be sufficient to induce a short telomere cellular response (38). In clinical settings, the average lymphocyte TL is measured, and it is usually below the tenth age-adjusted percentile in most patients with telomere-mediated disease (9). Therefore, while telomeres shorten with aging in $\mathrm{T}$ cells, the consequences of the resultant change may not always be sufficient to drive telomere-mediated phenotypes. Our data suggest that there are other telomere-independent $\mathrm{T}$ cell-aging programs that can provoke the $\mathrm{T}$ cell-aging phenotype. Understanding the predominant T cell-aging-driving mechanism for a given patient at the bedside and its genetic determinants is likely to have precision medicine applications, given the increasing use of T cell-directed therapies in cancer and autoimmunity. 
We focused our studies on young patients with short telomeres to uncouple the short telomere defect from those associated with aging in OA with normal TL. Our studies would, however, predict that OA with short TL may be prone to an even more severe immune aging phenotype that is driven by both the short telomere defect and additional changes that promote TL-independent $\mathrm{T}$ cell aging (Figure 5J). Indeed, there are clinical observations that support this idea. For example, short telomere OA are prone to developing lung disease that manifests as IPF. These IPF patients are exquisitely sensitive to developing fatal toxicities of $\mathrm{T}$ cell cytotoxic drugs, as has been recently shown (39). Moreover, IPF lung transplant recipients have higher rates of CMV infection than age-matched controls $(40,41)$. Our data indicate that reliable TL measurement, which is clinically available (9), is an independent predictor of T cell-proliferative potential, since short telomere-mediated $\mathrm{T}$ cell dysfunction lacks the classic immunophenotype associated with $\mathrm{T}$ cell aging. There are indeed data that support this view, and short TL is associated with lower antitumor proliferative responses and poor clinical outcomes in patients with melanoma who receive autologous $\mathrm{T}$ cell therapies (42). In summary, our data show that abnormally short telomeres cause a severe $\mathrm{T}$ cell immunodeficiency, but indicate their role in mediating $\mathrm{T}$ cell aging may be qualified and limited to only a subset of the population.

\section{Methods}

Subjects and controls. Patients were recruited from 2005 to 2015 as part of the Johns Hopkins Telomere Syndrome Registry (16, 43). Clinical data were extracted from existing medical records. Mutations were identified by PCR amplification followed by Sanger sequencing $(37,44)$, TruSeq Custom Amplicon sequencing followed by PCR and Sanger verification (45), or exome and genome sequencing (46-48). Healthy controls were recruited from volunteers in the Baltimore area from 2009 to 2014. Peripheral blood mononuclear cells (PBMCs) were separated by Ficoll-Paque from whole blood (SepMate, StemCell Technologies) and frozen until analysis. TL was measured by flow cytometry and FISH (flowFISH), as previously described $(9,49)$.

Sample preparation for immunophenotyping and antibodies. After thawing, PBMCs were treated to lyse contaminating red cells (Red Cell Lysis Buffer, eBioscience), then washed and suspended in $100 \mu \mathrm{l}$ $2 \% \mathrm{FBS} / \mathrm{PBS}$ solution for antibody staining. Viability was determined using LIVE/DEAD Fixable Stain conjugated with aqua fluorescent reactive dye (Invitrogen). Samples were then washed and suspended in $100 \mu \mathrm{l} 2 \% \mathrm{FBS} / \mathrm{PBS}$ for antibody staining. Antibody staining was performed for 20 minutes in the dark at $4^{\circ} \mathrm{C}$. Cell-surface phosphatidylserine (PS) was detected with APC-labeled annexin V in binding buffer (BD Biosciences) and counterstained with propidium iodide (PI) (BD Biosciences). Details regarding the human $(n=26)$ and mouse $(n=18)$ antibodies, including their conjugates, clone identifiers, and suppliers, are given in Supplemental Tables 5 and 6, respectively.

Flow cytometry and analysis. Flow cytometry data were acquired using CellQuest Pro v.5.1.1 on a FACSCalibur and using the FACSDiva v.6.1.2 on an LSR II (BD Biosciences). Ideal antibody concentrations were determined by serial dilutions and stain-index calculations. Single-color controls were collected for each antibody, and additional fluorescence-minus-one (FMO) controls were collected for low abundance markers (human PD-1, CCR7). Analyses were performed using FlowJo (v.X, Treestar). Both Boolean and sequential gating strategies were used to analyze the data. A lymphocyte gate was created on forward- versus side-scatter plots, followed by doublet and nonviable event exclusion gating. Subpopulations $\mathrm{CD}^{+} \mathrm{CD}^{+}$and $\mathrm{CD} 4^{+} \mathrm{CD}^{+}$ were then analyzed for markers of interest. At least 20,000 viable events were collected for each sample.

Cell sorting. For the TCR- $\beta$ sequencing studies, PBMCs were sorted using a MoFlo Legacy (Beckman Coulter). The sorted populations $\mathrm{CD}^{+}{ }^{+} \mathrm{CD} 4^{+} \mathrm{CD}^{-} 6^{-}$and $\mathrm{CD}^{+}{ }^{+} \mathrm{CD} 8^{+} \mathrm{CD}^{-} 6^{-}$(100,000 cells) were prepared for DNA extraction using the Gentra Puregene Blood Core Kit (QIAGEN). For the gene expression microarray, an average of 20,000 $\mathrm{CD}^{+}{ }^{+} \mathrm{CD} 8^{+} \mathrm{CD} 45 \mathrm{RA}^{+} \mathrm{CCR7} 7^{-}$(TEMRA) cells were sorted from PBMCs, using a MoFlo XPD (Beckman Coulter), directly into TRIzol (Invitrogen) and stored at $-80^{\circ} \mathrm{C}$. Total RNA was extracted using the Agencourt RNAdvance Tissue Kit (Beckman Coulter).

TREC assay. TRECs were measured by quantitative PCR (Cellular and Molecular Immunology Lab, Mayo Clinic) as previously described (22), using T cell DNA derived from freshly collected blood samples. $\mathrm{T}$ cells were isolated from EDTA whole blood using the RosetteSep (StemCell Technologies) for adult samples, while pediatric samples were enriched for total lymphocytes (StemCell Technologies) due to limited volume. Absolute $\mathrm{CD}^{+}, \mathrm{CD}^{+}$, and $\mathrm{CD}^{+}{ }^{+} \mathrm{T}$ cells were quantified in whole blood by flow cytometry. Quantitative real time PCR was performed using probes specific to signal-joint TRECs (sjTRECs), and the albumin gene was used for normalization of cell counts and as an internal control. TREC copy number was determined using standard curves, and the data were expressed as copies per 1 million $\mathrm{CD}^{+} \mathrm{T}$ cells. Age-appropriate reference ranges were generated from pediatric $(n=138)$ and adult $(n=116)$ controls, and values less than the fifth percentile were considered abnormal.

Mice. Mice were housed at the Johns Hopkins University School of Medicine campus. $m \mathrm{TR}^{-/-}$mice on the $\mathrm{C} 57 \mathrm{BL} / 6 \mathrm{~J}$ background were derived and maintained as previously described (23). WT donor mice were purchased from Charles River Laboratories or the Jackson Laboratory (B6.SJL-Ptprca Pepcb/BoyJ, males, 6 weeks, CD45.1). Recipient mice for adoptive transfer experiments were purchased from the Jackson Laboratory (C57BL/6J, 16 weeks, male and female, CD45.2). For complete blood counts, $100 \mu \mathrm{l}$ of blood from a cardiac puncture was analyzed on the Procyte Dx Hematology Analyzer (IDEXX Laboratories). Bone marrow and thymocytes were harvested using standard methods, and where relevant, samples were prepared and analyzed by flow cytometry, as described above.

Adoptive transfer experiments and thymocyte studies. Bone marrow from WT mice (CD45.1) was harvested from femurs and tibias and brought to single-cell suspension in $2 \% \mathrm{FBS} / \mathrm{PBS}$ supplemented with $1 \mathrm{mM}$ EDTA. For experiments testing the competency of the short telomere niche, hematopoietic progenitors were enriched by negative selection using the EasySep Mouse Hematopoietic Progenitor Cell Isolation Kit (StemCell Technologies). Enriched progenitors $\left(9 \times 10^{4}\right)$ were injected via tail vein into 18- to 22-week-old WT or $m T R^{-1-G 4}$ recipients (CD45.2) after a sublethal dose of irradiation (1 Gy, Cs-137 irradiator); this was modified from the protocol previously published (24). Recipient mice were sacrificed at preplanned time points, and the donor-derived fraction in the blood, thymus, and bone marrow were calculated by flow cytometry using antibodies specific for CD45 haplotypes (CD45.1 and CD45.2). At least 15,000 cells were analyzed for each sample. For experiments testing the competency of short telomere engraftment, 50,000 lineage-depleted hematopoeitic progenitors were 
injected into either WT or $m T R^{-/-G} 4$ recipient mice that were lethally irradiated (10 Gy). Engraftment for these experiments was assessed similarly, but from peripheral blood samples that were collected from the tail vein. Thymuses were harvested and dissociated mechanically using a 0.7 micron filter and syringe, then prepared for flow cytometry.

Mouse $\mathrm{T}$ cell apoptosis studies. Mouse $\mathrm{CD}^{+} \mathrm{T}$ cells were stimulated as described for human $\mathrm{T}$ cells (described below), except using mouse antibodies (Dynabeads Mouse T-Activator CD3/CD28, Life Technologies). Cell numbers and apoptosis fractions were then analyzed as outlined for the human studies. At least 15,000 cells were analyzed for each sample.

$T$ cell stimulation and proliferation. $\mathrm{CD}^{+} \mathrm{T}$ cells were isolated from PBMCs (EasySep Human T Cell Enrichment Kit, StemCell Technologies). Cells were resuspended in media in flat-bottomed 96-well plates (Corning) at $8 \times 10^{4}$ cells per well. Anti-CD3 and anti-CD28 covalently coupled to magnetic beads at a ratio of 1:1 were used for stimulation (Dynabeads Human T-Activator CD3/CD28, Life Technologies). The viable cell count was measured using the colorimetric WST assay (Dojindo Molecular Technologies), and cell death was analyzed using annexin/PI staining. Cell proliferation was measured by EdU staining using the Click-iT EdU Imaging Kit following kit instructions (Thermo Fisher Scientific). Briefly, cells were incubated for 12 hours with a final concentration of $1 \mu \mathrm{g} / \mu \mathrm{l} \mathrm{EdU}$. After incubation, cells were washed, stained with extracellular antibodies, and then fixed and permeabilized. Then cells were treated with the Click-iT reaction cocktail, washed, and examined by flow cytometry. A total of 15,000 events were collected for each sample.

Gene expression microarray of $C D 8^{+}$TEMRAs. RNA was amplified from sorted $\mathrm{CD} 8^{+} \mathrm{T}$ cells ( $\geq 98 \%$ purity) and reverse-transcribed using the Ovation Pico WTA System V2 (NuGEN). Transcriptional profiling was performed at the Johns Hopkins University Deep Sequencing and Microarray Core Facility using Affymetrix GeneChip PrimeView Human Gene Expression Array. CEL file data were extracted and normalized with the Partek Genomics Suite software using the robust multi-array average (RMA) algorithm. To ensure better understanding of the transcriptome, the array's Affy probe set annotation was updated to contemporary HUGO/NCBI nomenclature and those that could not be aligned to the genome were excluded.

Gene expression analysis. An ANOVA including all 3 groups generated $P$ values to select informative genes for a supervised heatmap construction. All the genes' $\log _{2}$ expression values were mean subtracted across all samples and imported into the Spotfire DecisionSite with Functional Genomics platform (TIBCO Spotfire) for further analyses. These mean subtracted expression values from 3,156 genes passing a $P<0.05$ cutoff ( 2 -sided) underwent hierarchical clustering with a correlation similarity measure and unweighted pair group method with arithmetic mean (UPGMA) (unweighted average) clustering method across all 12 samples assayed. The resulting heatmap was color coded by each gene's $\log _{2}$-fold difference from its average expression across all samples, i.e., its mean subtracted value.

Student's $t$ test was used to compare transcript expression levels between short telomere patients and YCs, and separately, OAs and YCs. The 48,880 aligned transcripts with gene-level annotation were selected for further analysis. A 2-sided $P$ value of less than 0.05 was considered significant. These genes' $\log _{2}$-fold change distribution was then evaluated to determine their SD higher or lower than the mean of 0 (i.e., no change). These fold change-based thresholds were then used to select the genes that were input for downstream functional pathway analysis.

Pathway analysis. To examine the pathways involved, we identified the genes with greater than 2 SD change in a given comparison. They were considered to have significant differential expression. This $2 \mathrm{SD}$ corresponded to a linear fold change of approximately \pm 2 .61-fold for ST versus YC, and 2.10 for OA versus YC ANOVAs, which resolved to 962 up- and 2,131 downregulated transcripts for the former, and 1,760 up- and 1,386 downregulated for the latter. These probe set transcripts were then uploaded to the IPA platform (IPA, QIAGEN, www.ingenuity.com) to evaluate their functional relevance in canonical pathways. The analysis was last performed on January 2, 2015. $P$ values for pathway selection were calculated using Fisher's exact test, and the top 20 pathways were analyzed for each 2-way comparison (Supplemental Tables 3 and 4). All the pathways listed had $P$ values of less than 0.01 (right-tailed). The MIAME-compliant microarray data were deposited in the NCBI's Gene Expression Omnibus database (GEO GSE77525).

TCR-V $\beta$ diversity by flow cytometry. Analysis of TCR-V $\beta$ diversity was performed by flow cytometry using the IOTest Beta Mark Kit (Beckman Coulter), which targets $24 \mathrm{~V} \beta$ proteins as previously described (28). The details for the $\mathrm{CD}^{+} \mathrm{APC}, \mathrm{CD} 4^{+}$PerCP Cy5.5, and $\mathrm{CD}^{+} \mathrm{V} 450$ antibodies used to distinguish the $24 \mathrm{~V} \beta$ family expression on $\mathrm{T}$ cell subsets are listed in Supplemental Table 5. At least 80,000 lymphocyte events were analyzed for each sample.

Sequencing of the TCR- $\beta$ locus. The TCR- $\beta$ CDR3 was sequenced using the immunoSEQ platform (Adaptive Biotechnologies), as previously described (29). We sequenced DNA from sorted CD8 ${ }^{+} \mathrm{T}$ cells. The immunoSEQ data calculated a comparable number of $\mathrm{CD} 8^{+} \mathrm{T}$ cells analyzed across groups with a mean of 8,335 cells and an average depth of coverage of $\times 31$. Several measures were used to examine the repertoire diversity. Richness was defined as the number of unique, productive (i.e., in-frame, no stop codon) sequences per sampled T cell. To compare the diversity of $\mathrm{V} \beta$ gene usage, the average frequency of gene usage was measured by group for each of the $58 \mathrm{~V} \beta$ genes detected. We calculated Pielou's J index as a measure of the diversity of gene usage (30).

Western blotting and qRT-PCR. Western blotting was performed as previously described (48). Briefly, mouse splenocytes were harvested on ice and $\mathrm{T}$ cells isolated as mentioned. For irradiated controls, mouse or splenocyte suspensions were irradiated (Cs-137). Cells were lysed using radioimmunoprecipitation assay (RIPA) buffer supplemented with a protease inhibitor cocktail (Roche). Proteins were resolved with SDS-PAGE and, after transfer to a PVDF membrane, blocked in 5\% milk in TBS-Tween. The following primary antibodies were used: Kap1 (rabbit, catalog A300-274, 1:1000; Bethyl Laborato-

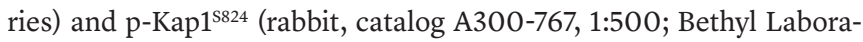
tories) with acting loading control (mouse, catalog ab8226, 1:2000; Abcam). Secondary antibodies were conjugated to dyes IR680 (donkey) or IR800 (goat) (1:10,000; LI-COR), and blots were visualized using an Odyssey scanner (LI-COR). Quantitative reverse-transcription PCR (qRT-PCR) on total RNA isolated from T cells was performed as previously described using iQSYBR Green Supermix (Bio-Rad) (50, 51). Amplifying primers were previously published: $\mathrm{p} 16$ and $\mathrm{p} 21$, and p27, Hprt (52), and Bax (51).

Statistics. We used GraphPad Prism v.6.05 (GraphPad Software) to generate the graphics and, unless otherwise noted, for statistical comparisons. Means were compared by Student's $t$ test and, where appropriate, Mann-Whitney $U$ test. All the $P$ values reported, unless other- 
wise noted, are 2-sided. We applied a correction for multiple testing (Benjamini-Hochberg procedure) (53) for the number of hypotheses tested, and Benjamini-Hochberg corrected values for a FDR of $0.05 \%$ are reported. $P$ values of less than 0.05 were considered significant. The statistical tests used in the gene expression microarray analyses are described separately above.

Study approval. The study was approved by the Johns Hopkins Medicine Institutional Review Board, and all the subjects gave written, informed consent. The consent form included permission to publish relevant images and photographs. Mice were housed in the East Baltimore campus of the Johns Hopkins University School of Medicine, and the study and its procedures were approved by the Institutional Animal Use and Care Committee.

\section{Author contributions}

LL and MA conceived the ideas and designed the experiments. CLW and VSH designed, performed, and analyzed the flow cytometry and sorting experiments. CGK gave input on the design of the human flow cytometry experiments. CLW and CCT analyzed the microarray data. CLW and RSA performed and analyzed the TREC studies. CLW and DH analyzed immunoSEQ data. DLG optimized and performed quantitative PCR and flow cytometry experiments. CDA obtained and analyzed the genetic and clinical data with CLW and MA. JS, JBJ, and JKA provided key reagents. SD designed mouse experiments and CLW performed them. JKA performed and analyzed mouse and TL experiments. CLW per- formed the other experiments. MA analyzed the clinical data and wrote the paper with input from CLW. All the authors reviewed and approved the manuscript.

\section{Acknowledgments}

We are grateful to all the subjects who volunteered for this study, their families, and their referring clinicians. We acknowledge helpful discussions with Biljana Horn. We acknowledge help from Margaret Strong from Carol Greider's lab in providing mice. We acknowledge support from these Johns Hopkins Cores: Genetics Center Resource Facilities (Melissa Olsen), the Human Systems Biology Center (Haiping Hao), the Bloomberg School of Public Health Flow Cytometry Core (Hao Zhang), and experimental advice from Xuhang Li. We are grateful to Lisa M. Nicholson for help with the statistical analyses and Catherine Kiefe from the Department of Art as Applied to Medicine for help with the figures. This work was supported by NIH grants R01CA160433, R01HL119476, and RO1CA225027 (to MA) and R01HL110907 (to LL), funds from the Maryland Stem Cell Research Foundation, the Commonwealth Foundation, the Gary Williams Foundation, the S\&R Foundation Kuno Award (to MA), and the Turock Scholar Fund (to the Telomere Center at Johns Hopkins).

Address correspondence to: Mary Armanios, 1650 Orleans St., CRB 1 Room 186, Baltimore, Maryland 21287, USA. Phone: 410.502.3817; Email: marmani1@jhmi.edu.
1. Wherry EJ, Kurachi M. Molecular and cellular insights into T cell exhaustion. Nat Rev Immunol. 2015;15(8):486-499.

2. Goronzy JJ, Weyand CM. Understanding immunosenescence to improve responses to vaccines. Nat Immunol. 2013;14(5):428-436.

3. Armanios M, Alder JK, Parry EM, Karim B, Strong MA, Greider CW. Short telomeres are sufficient to cause the degenerative defects associated with aging. Am J Hum Genet. 2009;85(6):823-832.

4. Codd V, et al. Identification of seven loci affecting mean telomere length and their association with disease. Nat Genet. 2013;45(4):422-427e1.

5. Aviv A. Genetics of leukocyte telomere length and its role in atherosclerosis. Mutat Res. 2012;730(1-2):68-74.

6. d'Adda di Fagagna F, et al. A DNA damage checkpoint response in telomere-initiated senescence. Nature. 2003;426(6963):194-198.

7. Chin L, et al. p53 deficiency rescues the adverse effects of telomere loss and cooperates with telomere dysfunction to accelerate carcinogenesis. Cell. 1999;97(4):527-538.

8. Hemann MT, Strong MA, Hao LY, Greider CW. The shortest telomere, not average telomere length, is critical for cell viability and chromosome stability. Cell. 2001;107(1):67-77.

9. Alder JK, et al. Diagnostic utility of telomere length testing in a hospital-based setting. Proc Natl Acad Sci U S A. 2018;115(10):E2358-E2365.

10. Buchkovich KJ, Greider CW. Telomerase regulation during entry into the cell cycle in normal human T cells. Mol Biol Cell. 1996;7(9):1443-1454.

11. Rufer N, et al. Telomere fluorescence measurements in granulocytes and $\mathrm{T}$ lymphocyte subsets point to a high turnover of hematopoietic stem cells and memory T cells in early childhood. J Exp Med.1999;190(2):157-167.

12. Aubert G, Baerlocher GM, Vulto I, Poon SS, Lansdorp PM. Collapse of telomere homeostasis in hematopoietic cells caused by heterozygous mutations in telomerase genes. PLoS Genet. 2012;8(5):e1002696.

13. Stanley SE, Armanios M. The short and long telomere syndromes: paired paradigms for molecular medicine. Curr Opin Genet Dev. 2015;33:1-9.

14. Dokal I. Dyskeratosis congenita in all its forms. $\mathrm{Br}$ J Haematol. 2000;110(4):768-779.

15. Glousker G, Touzot F, Revy P, Tzfati Y, Savage SA. Unraveling the pathogenesis of HoyeraalHreidarsson syndrome, a complex telomere biology disorder. Br J Haematol. 2015;170(4):457-471.

16. Jonassaint NL, Guo N, Califano JA, Montgomery EA, Armanios M. The gastrointestinal manifestations of telomere-mediated disease. Aging Cell. 2013;12(2):319-323.

17. Stanley SE, Rao AD, Gable DL, McGrath-Morrow $\mathrm{S}$, Armanios M. Radiation sensitivity and radiation necrosis in the short telomere syndromes. Int J Radiat Oncol Biol Phys. 2015;93(5):1115-1117.

18. Yabe M, et al. Fatal interstitial pulmonary disease in a patient with dyskeratosis congenita after allogeneic bone marrow transplantation. Bone Marrow Transplant. 1997;19(4):389-392.

19. Kimmig S, et al. Two subsets of naive T helper cells with distinct $\mathrm{T}$ cell receptor excision circle content in human adult peripheral blood. J Exp Med. 2002;195(6):789-794.

20. Kwan A, et al. Newborn screening for severe combined immunodeficiency in 11 screen- ing programs in the United States. JAMA. 2014;312(7):729-738

21. Gaspar HB, Hammarström L, Mahlaoui N, Borte $\mathrm{M}$, Borte $\mathrm{S}$. The case for mandatory newborn screening for severe combined immunodeficiency (SCID). J Clin Immunol. 2014;34(4):393-397.

22. Douek DC, et al. Assessment of thymic output in adults after haematopoietic stem-cell transplantation and prediction of $\mathrm{T}$-cell reconstitution. Lancet. 2000;355(9218):1875-1881.

23. Blasco MA, et al. Telomere shortening and tumor formation by mouse cells lacking telomerase RNA. Cell. 1997;91(1):25-34.

24. Serwold T, Ehrlich LI, Weissman IL. Reductive isolation from bone marrow and blood implicates common lymphoid progenitors as the major source of thymopoiesis. Blood. 2009;113(4):807-815.

25. Wong KK, et al. Telomere dysfunction impairs DNA repair and enhances sensitivity to ionizing radiation. Nat Genet. 2000;26(1):85-88.

26. Choudhury AR, et al. Cdkn1a deletion improves stem cell function and lifespan of mice with dysfunctional telomeres without accelerating cancer formation. Nat Genet. 2007;39(1):99-105.

27. Hoffman ES, et al. Productive T-cell receptor beta-chain gene rearrangement: coincident regulation of cell cycle and clonality during development in vivo. Genes Dev. 1996;10(8):948-962.

28. Degauque N, Boeffard F, Foucher Y, Ballet C, Brouard S, Soulillou JP. The blood of healthy individuals exhibits CD8 T cells with a highly altered TCR Vb repertoire but with an unmodified phenotype. PLoS One. 2011;6(6):e21240.

29. Carlson CS, et al. Using synthetic templates to design an unbiased multiplex PCR assay. Nat 
Commun. 2013;4:2680.

30. Pielou EC. Species-diversity and patterndiversity in the study of ecological succession. J Theor Biol. 1966;10(2):370-383.

31. Qi Q, et al. Diversity and clonal selection in the human T-cell repertoire. Proc Natl Acad Sci U S A. 2014;111(36):13139-13144.

32. Kared H, Martelli S, Ng TP, Pender SL, Larbi A. CD57 in human natural killer cells and T-lymphocytes. Cancer Immunol Immunother. 2016;65(4):441-452.

33. Hao LY, Strong MA, Greider CW. Phosphorylation of H2AX at short telomeres in T cells and fibroblasts. J Biol Chem. 2004;279(43):45148-45154.

34. Knudson M, Kulkarni S, Ballas ZK, Bessler M, Goldman F. Association of immune abnormalities with telomere shortening in autosomaldominant dyskeratosis congenita. Blood. 2005;105(2):682-688.

35. Jyonouchi S, Forbes L, Ruchelli E, Sullivan KE. Dyskeratosis congenita: a combined immunodeficiency with broad clinical spectrum--a single-center pediatric experience. Pediatr Allergy Immunol. 2011;22(3):313-319.

36. Dietz AC, et al. Disease-specific hematopoietic cell transplantation: nonmyeloablative conditioning regimen for dyskeratosis congenita. Bone Marrow Transplant. 2011;46(1):98-104.

37. Parry EM, Alder JK, Qi X, Chen JJ, Armanios M. Syndrome complex of bone marrow failure and pulmonary fibrosis predicts germline defects in telomerase. Blood. 2011;117(21):5607-5611.

38. Baird DM, Rowson J, Wynford-Thomas D, Kipling D. Extensive allelic variation and ultrashort telomeres in senescent human cells. Nat Genet. 2003;33(2):203-207.

39. Raghu G, Anstrom KJ, King TE Jr, Lasky JA, Martinez FJ. Prednisone, azathioprine, and $\mathrm{N}$-acetylcysteine for pulmonary fibrosis. $\mathrm{N} \mathrm{Engl} \mathrm{J}$ Med. 2012;366(21):1968-1977.

40. Silhan LL, et al. Lung transplantation in telomerase mutation carriers with pulmonary fibrosis. Eur Respir J. 2014;44(1):178-187.

41. Popescu I, et al. Impaired CMV immunity in idiopathic pulmonary fibrosis lung transplant recipients with short telomeres [published online ahead of print August 8, 2010]. Am J Respir Crit Care Med. https://doi.org/10.1164/rccm.201805-0825OC.

42. Rosenberg SA, et al. Durable complete responses in heavily pretreated patients with metastatic melanoma using T-cell transfer immunotherapy. Clin Cancer Res. 2011;17(13):4550-4557.

43. Gorgy AI, et al. Hepatopulmonary syndrome is a frequent cause of dyspnea in the short telomere disorders. Chest. 2015;148(4):1019-1026.

44. Parry EM, et al. Decreased dyskerin levels as a mechanism of telomere shortening in $\mathrm{X}$-linked dyskeratosis congenita. JMed Genet. 2011;48(5):327-333.

45. Stanley SE, et al. Telomerase mutations in smokers with severe emphysema. JClin Invest. 2015;125(2):563-570

46. Alder JK, et al. Telomere phenotypes in females with heterozygous mutations in the dyskeratosis congenita 1 (DKC1) gene. Hum Mutat. 2013;34(11):1481-1485.

47. Alder JK, Stanley SE, Wagner CL, Hamilton M, Hanumanthu VS, Armanios M. Exome sequencing identifies mutant TINF2 in a family with pulmonary fibrosis. Chest. 2015;147(5):1361-1368.

48. Stanley SE, et al. Loss-of-function mutations in the RNA biogenesis factor NAF1 predispose to pulmonary fibrosis-emphysema. Sci Transl Med. 2016;8(351):351ra107.

49. Baerlocher GM, Vulto I, de Jong G, Lansdorp PM. Flow cytometry and FISH to measure the average length of telomeres (flow FISH). Nat Protoc. 2006;1(5):2365-2376.

50. Guo N, et al. Short telomeres compromise $\beta$-cell signaling and survival. PLoS One. 2011;6(3):e17858.

51. Alder JK, et al. Telomere dysfunction causes alveolar stem cell failure. Proc Natl Acad Sci U S A. 2015;112(16):5099-5104.

52. Alder JK, et al. Telomere length is a determinant of emphysema susceptibility. Am J Respir Crit Care Med. 2011;184(8):904-912.

53. Benjamini Y, Hochberg Y. Controlling the false discovery rate: a practical and powerful approach to multiple testing. J R Stat Soc Series B Stat Methodol. 1995;57(1):289-300. 